\title{
Numerical methods for
} immersed FSI with thin-walled structures

Ludovic Boilevin-Kayl, Miguel A. Fernández, Jean-Frédéric Gerbeau

\section{RESEARCH}

REPORT

$\mathrm{N}^{\circ} 9151$

February 2018

Project-Team REO 



\title{
Inzía
}

\section{Numerical methods for immersed FSI with thin-walled structures}

\author{
Ludovic Boilevin-Kay閶, Miguel A. Fernández*†, \\ Jean-Frédéric Gerbeau*† \\ Project-Team REO \\ Research Report n 9151 - February 2018 - 37pages
}

\begin{abstract}
The numerical simulation of a thin-walled structure immersed in an incompressible fluid can be addressed by various methods. In this paper, three of them are considered: the Arbitrary Lagrangian-Eulerian (ALE) method, the Fictitious Domain/Lagrange multipliers (FD) method and the Nitsche-XFEM method. Taking ALE as a reference, the advantages and limitations of FD and Nitsche-XFEM are carefully discussed on three benchmark test cases which have been chosen to be representative of typical difficulties encountered in valves or living cells simulations.
\end{abstract}

Key-words: fluid-structure interaction, incompressible fluid, immersed thin-walled structure, unfitted meshes, fitted meshes, fictitious domain method, XFEM method, Nitsche method, ALE method.

* Inria Paris, 75012 Paris, France

† Sorbonne Université, Lab. Jacques-Louis Lions, 75252 Paris, France

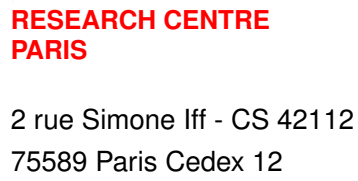




\section{Méthodes numériques pour l'IFS avec des structures minces immergés}

Résumé : La simulation numérique d'une structure mince immergée dans un fluide incompressible peut être abordée par différentes méthodes. Dans cet article, trois d'entre elles sont considérées: la méthode Arbitrairement Lagrangienne Eulérienne (ALE), la méthode de domaine fictif avec multiplicateurs de Lagrange (FD) et la méthode Nitsche-XFEM. En prenant la méthode ALE comme référence, les avantages et les limites des méthodes FD et Nitsche-XFEM sont soigneusement discutés sur trois cas tests qui ont été choisis pour être représentatifs des difficultés typiques rencontrées dans les simulations de valves ou de cellules.

Mots-clés : interaction fluide-structure, fluide incompressible, structure mince immergée, maillages non compatibles, maillages compatibles, méthode de domaine fictif, méthode XFEM, méthode de Nitsche, méthode ALE. 


\section{Introduction}

The numerical simulation of the mechanical interaction between an incompressible viscous fluid and an immersed thin-walled elastic structure is an essential ingredient in the mathematical modeling of many living systems. As examples, we can mention the opening and closing dynamics of heart valves, the wings of a bird interacting with the air or the fins of a fish moving in water (see, e.g., 34, 38, 48,).

The numerical methods for this type of fluid-structure interaction (FSI) problems are generally tailored to the amount of solid displacement within the fluid. Problems with low or moderate interface deflections can be successfully simulated using fitted (moving) mesh techniques, based on an arbitrary Lagrangian-Eulerian (ALE) description of the fluid. However, for problems involving large structural displacements (with solids that might come into contact or that might break up) the ALE formalism becomes cumbersome. A favored alternative is the combination of an Eulerian formalism in the fluid with an unfitted mesh discretization, in which the fluidstructure interface deforms independently of a background fluid mesh. Among these approaches, we can mention the Immersed Boundary method (IB) (see, e.g., 40, 9), and methods with Lagrange multipliers, that will be called Fictitious Domain methods (FD) for simplicity (see, e.g., [28, 4, 20, 3, 10, 11]), which keep a Lagrangian formalism for the solid, and the methodologies based on a fully Eulerian formalism for the fluid (see, e.g., [19, 43]).

In general, these unfitted mesh approaches are known to be inaccurate in space, because the discrete treatment of the interface conditions and/or because the fluid spatial discretization do/does not allow for (weak and strong) discontinuities across the interface. Mesh adaptation mitigates these issues (see, e.g., [30]), but it does not cure the problem. The extended-FEM (XFEM) method, which combines a cut-FEM methodology with a local enrichment (see, e.g., [54, 26, 45]), overcomes these issues but at the price of introducing additional unknowns (Lagrange multipliers) and a degradation of robustness (with respect to the interface cuts). These difficulties have been recently circumvented by the Nitsche-XFEM method reported in [2, 13, which combines a Nitsche's treatment of the interface coupling with overlapping meshes and a suitable stabilization on the interfacial zone. The superior accuracy properties of cut-FEM approaches comes however at a price: these methods demand a much more involved computer implementation and require a specific evaluation of the interface intersections, which can be computationally challenging (notably in 3D, for arbitrary unstructured meshes).

Unfitted mesh methods for FSI are rarely compared between them in the literature. It is hence unclear to what extend cut-FEM methodologies are appealing in practice, with respect to more standard IB or FD methods. The first contribution of this paper consists in providing insight into this problem. To this purpose, we compare and validate some of the aforementioned unfitted mesh methods in a series of 2D-FSI benchmarks with dynamics interfaces undergoing large deflections. We consider an archetypal sample of unfitted mesh methods: the FD method as implemented in [21, an enhanced variant from 34] and the Nitsche-XFEM method introduced in 2. The numerical approximation provided by an ALE fitted mesh approach is used as reference for the comparisons. The second contribution of this work has to do with the above referenced unfitted Nitsche-XFEM method, which is here formulated and validated, for the first time, in a fully non-linear framework, involving a general thin-wall solid model for large displacements and rotations. The numerical results reported in [2] were limited to moderate (normal) displacements using a simple string model.

The rest of the paper is organized as follows. Section 2 presents the different mathematical models and formulations considered through the paper. The numerical methods are described in Section 3. Section 4 presents the comparisons of the results obtained with the three FSI benchmarks tests. Finally, a summary of the main conclusions is given in Section 5 . 


\section{Problem setting and mathematical formulations}

In this section we discuss the mathematical models considered through the paper. The fluid is modeled by the incompressible Navier-Stokes equations in the Eulerian or in the ALE (Arbitrary Lagrangian-Eulerian) formalisms. The thin-walled solid is described by a non-linear beam model in Lagrangian form.

\subsection{Geometric configurations and notation}

We consider a fluid-structure interaction problem coupling an incompressible viscous fluid with an immersed thin-walled solid. Let $\Sigma \subset \mathbb{R}^{2}$ be the reference configuration of the solid mid-surface. The current position of the interface, denoted by $\Sigma(t)$, is given in terms of the deformation map $\phi: \Sigma \times \mathbb{R}^{+} \longrightarrow \mathbb{R}^{2}$ as $\Sigma(t)=\boldsymbol{\phi}(\Sigma, t)$, with $\boldsymbol{\phi} \stackrel{\text { def }}{=} \boldsymbol{I}_{\Sigma \times \mathbb{R}^{+}}+\boldsymbol{d}$ where $\boldsymbol{d}$ denotes the displacement of the solid. The structure is supposed to move within a fixed domain $\Omega \subset \mathbb{R}^{2}$ with boundary

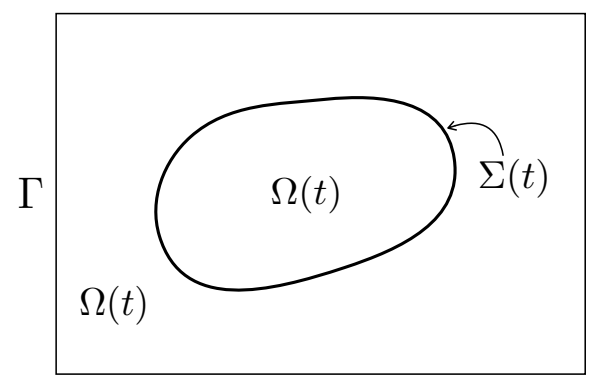

Figure 1: The computational domain $\Omega$.

$\Gamma \stackrel{\text { def }}{=} \partial \Omega$ (see Figure 1). The fluid occupies the time-dependent domain

$$
\Omega(t) \stackrel{\text { def }}{=} \Omega \backslash \Sigma(t) \subset \mathbb{R}^{2},
$$

with its boundary being partitioned as $\partial \Omega(t)=\Sigma(t) \cup \Gamma$. The interface $\Sigma(t)$ is assumed to be oriented by a unit surface normal vector field denoted by $\boldsymbol{n}_{\Sigma}$. We can hence define positive and negative sides on $\Sigma(t)$, with respective unit normals $\boldsymbol{n}_{1} \stackrel{\text { def }}{=} \boldsymbol{n}_{\Sigma}$ and $\boldsymbol{n}_{2} \stackrel{\text { def }}{=}-\boldsymbol{n}_{\Sigma}$. For a given continuous field $f$ defined in $\Omega$ (possibly discontinuous across the interface), we define its sided-restrictions to $\Sigma(t)$, denoted by $f_{1}$ and $f_{2}$, as

$$
f_{i}(\boldsymbol{x}) \stackrel{\text { def }}{=} \lim _{\xi \rightarrow 0^{+}} f\left(\boldsymbol{x}+\xi \boldsymbol{n}_{i}\right), \quad i=1,2,
$$

for all $\boldsymbol{x} \in \Sigma(t)$, and the following jump and average operators across $\Sigma(t)$ :

$$
\llbracket f \rrbracket \stackrel{\text { def }}{=} f^{+}-f^{-}, \quad \llbracket f \boldsymbol{n} \rrbracket \stackrel{\text { def }}{=} f_{1} \boldsymbol{n}_{1}+f_{2} \boldsymbol{n}_{2}, \quad\{f\} \stackrel{\text { def }}{=} \frac{1}{2}\left(f^{+}+f^{-}\right) .
$$

Finally, in order to ease the presentation, we introduce the following notation $\phi_{t} \stackrel{\text { def }}{=} \phi(\cdot, t)$, for all field $\phi$ defined in $\Omega \times \mathbb{R}^{+}$.

\subsection{Solid model}

We consider a non-linear Reissner-Mindlin beam model with a reference configuration given by the mid-surface $\Sigma$. The beam kinematics are described by the displacement of the mid-surface $\boldsymbol{d}$ 
and by a field of director vectors $\boldsymbol{a}$ in the deformed configuration. These unit vectors correspond to the material lines in the reference configuration. We recall that Reissner-Mindlin kinematics assume that such material lines, originally aligned with $\boldsymbol{n}_{\Sigma}$, remain straight and do not stretch during the deformation (see, e.g., 6, 17]).

In this framework, the equilibrium of the solid is described by the following non-linear problem: find the solid mid-surface displacement $\boldsymbol{d}: \Sigma \times \mathbb{R}^{+} \rightarrow \mathbb{R}^{2}$, the solid mid-surface velocity $\dot{\boldsymbol{d}}: \Sigma \times \mathbb{R}^{+} \rightarrow \mathbb{R}^{2}$ and the director vector $\boldsymbol{a}: \Sigma \times \mathbb{R}^{+} \rightarrow \mathbb{R}^{2}$ with unit length, $|\boldsymbol{a}|=1$, such that

$$
\left\{\begin{array}{rcc}
\rho^{\mathrm{s}} \epsilon \partial_{t} \dot{\boldsymbol{d}}+\boldsymbol{L}_{\boldsymbol{d}}(\boldsymbol{d}, \boldsymbol{a})=\boldsymbol{T} & \text { on } & \Sigma, \\
\boldsymbol{L}_{\boldsymbol{a}}(\boldsymbol{d}, \boldsymbol{a})=\mathbf{0} & \text { on } & \Sigma, \\
\dot{\boldsymbol{d}}=\partial_{t} \boldsymbol{d} & \text { on } & \Sigma,
\end{array}\right.
$$

hold for all $t>0$. Here, $\rho^{\mathrm{s}}$ stands for he solid density, $\boldsymbol{T}$ denotes a given source term and the abstract surface operators $\boldsymbol{L}_{\boldsymbol{d}}$ and $\boldsymbol{L}_{\boldsymbol{a}}$ describe the elastic behaviour of the beam, with $\boldsymbol{L}_{\boldsymbol{a}}(\boldsymbol{d}, \boldsymbol{a})=\mathbf{0}$ representing, in particular, the bending moments and shear stresses equilibrium (rotational inertia effects are neglected).

\subsection{Coupled problem with Eulerian formalism in the fluid}

The first considered non-linear coupled problem reads as follow: find the fluid velocity and pressure $\boldsymbol{u}: \Omega \times \mathbb{R}^{+} \rightarrow \mathbb{R}^{2}, p: \Omega \times \mathbb{R}^{+} \rightarrow \mathbb{R}$, the solid displacement and velocity $\boldsymbol{d}: \Sigma \times \mathbb{R}^{+} \rightarrow \mathbb{R}^{2}$, $\dot{\boldsymbol{d}}: \Sigma \times \mathbb{R}^{+} \rightarrow \mathbb{R}^{2}$ and the unit director vector $\boldsymbol{a}: \Sigma \times \mathbb{R}^{+} \rightarrow \mathbb{R}^{2}$ such that

$$
\begin{gathered}
\left\{\begin{array}{r}
\rho^{\mathrm{f}} \partial_{t} \boldsymbol{u}+\rho^{\mathrm{f}} \boldsymbol{u} \cdot \nabla \boldsymbol{u}-\operatorname{div} \boldsymbol{\sigma}(\boldsymbol{u}, p)=\mathbf{0} \text { in } \quad \Omega(t), \\
\operatorname{div} \boldsymbol{u}=0 \quad \text { in } \quad \Omega(t), \\
\boldsymbol{u}=\mathbf{0} \text { on } \quad \Gamma,
\end{array}\right. \\
\left\{\begin{array}{r}
\rho^{\mathrm{s}} \epsilon \partial_{t} \dot{\boldsymbol{d}}+\boldsymbol{L}_{\boldsymbol{d}}(\boldsymbol{d}, \boldsymbol{a})=\boldsymbol{T} \text { on } \quad \Sigma, \\
\boldsymbol{L}_{\boldsymbol{a}}(\boldsymbol{d}, \boldsymbol{a})=\mathbf{0} \text { on } \Sigma, \\
\dot{\boldsymbol{d}}=\partial_{t} \boldsymbol{d} \text { on } \Sigma,
\end{array}\right. \\
\left\{\begin{array}{r}
\boldsymbol{\phi}=\boldsymbol{I}_{\Sigma \times \mathbb{R}^{+}}+\boldsymbol{d}, \quad \Sigma(t)=\boldsymbol{\phi}(\Sigma, t), \quad \Omega(t)=\Omega \backslash \Sigma(t), \\
\boldsymbol{u}=\dot{\boldsymbol{d}} \circ \boldsymbol{\phi}_{t}^{-1} \quad \text { on } \Sigma(t), \\
\int_{\Sigma} \boldsymbol{T} \cdot \boldsymbol{y}=-\int_{\Sigma(t)} \llbracket \boldsymbol{\sigma}(\boldsymbol{u}, p) \boldsymbol{n} \rrbracket \cdot \boldsymbol{y} \circ \boldsymbol{\phi}_{t}^{-1},
\end{array}\right.
\end{gathered}
$$

for all smooth test function $\boldsymbol{y}: \Sigma \rightarrow \mathbb{R}^{2}$. Here, the symbol $\rho^{\mathrm{f}}$ denotes the fluid density and the fluid Cauchy stress tensor is given by

$$
\boldsymbol{\sigma}(\boldsymbol{u}, p) \stackrel{\text { def }}{=} 2 \mu \boldsymbol{\epsilon}(\boldsymbol{u})-p \boldsymbol{I}, \quad \boldsymbol{\epsilon}(\boldsymbol{u}) \stackrel{\text { def }}{=} \frac{1}{2}\left(\nabla \boldsymbol{u}+\nabla \boldsymbol{u}^{\mathrm{T}}\right),
$$

where $\mu$ denotes the fluid dynamic viscosity

The three relations in (3) enforce, respectively, the geometric, the kinematic and the dynamic interface coupling. Note that we fully identify the solid mid-surface with the fluid-structure interface, by neglecting all the beam thickness effects in the interface coupling. This is a commonly used simplification when coupling thin-walled and thick-walled media (see, e.g., 18). Note that the coupled problem (1)-(3) has an inherent mixed Eulerian-Lagrangian nature, which is a direct consequence of the presence of the maps $\phi_{t}$ and $\phi_{t}^{-1}$ in the interface coupling (3). This introduces additional (geometrical) non-linearities. 


\subsection{Coupled problem with ALE formalism in the fluid}

The moving control volume $\Omega(t)$ is assumed to be parametrizable as $\Omega(t)=\mathcal{A}(\Omega, t)$, where $\mathcal{A}: \Omega \times \mathbb{R}^{+} \rightarrow \mathbb{R}^{2}$ is a one-to-one mapping given by the relation $\mathcal{A}=\boldsymbol{I}_{\Omega \times \mathbb{R}^{+}}+\boldsymbol{d}^{\mathrm{f}}$, in terms of the fluid domain displacement $\boldsymbol{d}^{\mathrm{f}}$ (see Figure 2).

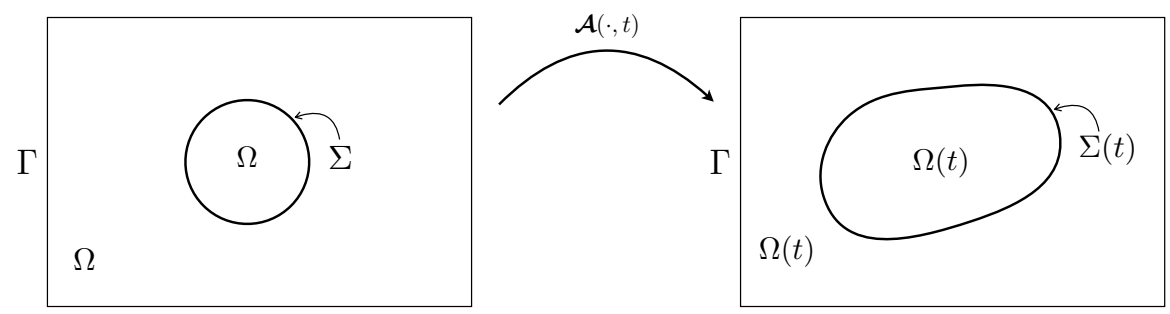

Figure 2: The ALE map.

The geometrical compatibility between the fluid and solid domains can hence be guaranteed through a relation of the type $\boldsymbol{d}^{\mathrm{f}}=\mathcal{L}(\boldsymbol{d})$, where $\mathcal{L}(\boldsymbol{d})$ represents a suitable lifting of $\boldsymbol{d}$ from $\Sigma$ to $\Omega$ which vanishes on $\Gamma$. Note that $\boldsymbol{d}^{\mathrm{f}}$ can be arbitrarily defined in $\Omega \backslash \Sigma$. By a simple use of the chain rule, we have the following relation:

$$
\partial_{t} \boldsymbol{u}=\left.\partial_{t}\right|_{\mathcal{A}} \boldsymbol{u}-\boldsymbol{w} \cdot \boldsymbol{\nabla} \boldsymbol{u}
$$

where $\left.\partial_{t}\right|_{\mathcal{A}}$ denotes the ALE time derivative and $\boldsymbol{w} \stackrel{\text { def }}{=} \partial_{t} \mathcal{A}=\partial_{t} \boldsymbol{d}^{\mathrm{f}}$ the fluid domain velocity. The fundamental motivation of (4) is that it facilitates the time discretization of terms in $\Omega(t)$ involving quantities originally defined in $\Omega$ which are transported to $\Omega(t)$ through $\mathcal{A}$ (see Section 3.1).

Using the above relations, the coupled problem $(1)-(3)$ admits the following equivalent formulation: find the fluid domain displacement $\boldsymbol{d}^{\mathrm{f}}: \Omega \times \mathbb{R}^{+} \rightarrow \mathbb{R}^{2}$, the fluid velocity $\boldsymbol{u}: \Omega \times \mathbb{R}^{+} \rightarrow \mathbb{R}^{2}$, the fluid pressure $p: \Omega \times \mathbb{R}^{+} \rightarrow \mathbb{R}$, the solid mid-surface displacement $\boldsymbol{d}: \Sigma \times \mathbb{R}^{+} \rightarrow \mathbb{R}^{2}$, the solid mid-surface velocity $\dot{\boldsymbol{d}}: \Sigma \times \mathbb{R}^{+} \rightarrow \mathbb{R}^{2}$ and the unit director vector $\boldsymbol{a}: \Sigma \times \mathbb{R}^{+} \rightarrow \mathbb{R}^{2}$, such that

$$
\begin{aligned}
& \left\{\begin{aligned}
\left.\rho^{\mathrm{f}} \partial_{t}\right|_{\mathcal{A}} \boldsymbol{u}+\rho^{\mathrm{f}}(\boldsymbol{u}-\boldsymbol{w}) \cdot \nabla \boldsymbol{u}-\operatorname{div} \boldsymbol{\sigma}(\boldsymbol{u}, p)=\mathbf{0} & \text { in } \Omega(t), \\
\operatorname{div} \boldsymbol{u}=0 & \text { in } \Omega(t), \\
\boldsymbol{u}=\mathbf{0} & \text { on } \quad \Gamma,
\end{aligned}\right. \\
& \left\{\begin{array}{rlc}
\rho^{\mathrm{s}} \epsilon \partial_{t} \dot{\boldsymbol{d}}+\boldsymbol{L}_{\boldsymbol{d}}(\boldsymbol{d}, \boldsymbol{a})=\boldsymbol{T} & \text { on } & \Sigma, \\
\boldsymbol{L}_{\boldsymbol{a}}(\boldsymbol{d}, \boldsymbol{a})=\mathbf{0} & \text { on } & \Sigma, \\
\dot{\boldsymbol{d}}=\partial_{t} \boldsymbol{d} & \text { on } & \Sigma,
\end{array}\right. \\
& \left\{\begin{array}{l}
\boldsymbol{d}^{\mathrm{f}}=\mathcal{L}(\boldsymbol{d}), \quad \boldsymbol{w}=\partial_{t} \boldsymbol{d}^{\mathrm{f}}, \quad \mathcal{A}=\boldsymbol{I}_{\Omega \times \mathbb{R}^{+}}+\boldsymbol{d}^{\mathrm{f}}, \quad \Omega(t)=\mathcal{A}(\Omega, t), \\
\boldsymbol{u}=\dot{\boldsymbol{d}} \circ \mathcal{A}_{t}^{-1} \quad \text { on } \quad \Sigma(t), \\
\int_{\Sigma} \boldsymbol{T} \cdot \boldsymbol{y}=-\int_{\Sigma(t)} \llbracket \boldsymbol{\sigma}(\boldsymbol{u}, p) \boldsymbol{n} \rrbracket \cdot \boldsymbol{y} \circ \mathcal{A}_{t}^{-1},
\end{array}\right.
\end{aligned}
$$

for all smooth function $\boldsymbol{y}: \Sigma \rightarrow \mathbb{R}^{2}$. 


\section{Numerical methods}

Though the coupled problems (1)-(3) and (5)-(7) are equivalent at the continuous level, their discretization generally leads to different numerical approaches.

In what follows, $\tau>0$ denotes the time-step length, $t_{n} \stackrel{\text { def }}{=} n \tau$ for $n \in \mathbb{N}$, and $\partial_{\tau} x^{n} \stackrel{\text { def }}{=}$ $\frac{1}{\tau}\left(x^{n}-x^{n-1}\right)$ stands for the first-order backward difference. We will also make use of the standard Sobolev spaces $H^{m}(\omega)(m \geq 0)$. The closed subspaces $H_{\Gamma}^{1}(\omega)$, of functions in $H^{1}(\omega)$ with zero trace on $\Gamma$, and $L_{0}^{2}(\omega)$, of functions in $L^{2}(\omega)$ with zero mean in $\omega$, will also be used. The scalar product in $L^{2}(\omega)$ is denoted by $(\cdot, \cdot)_{\omega}$. We consider $\boldsymbol{V} \stackrel{\text { def }}{=}\left[H_{\Gamma}^{1}(\Omega)\right]^{2}$ and $Q \stackrel{\text { def }}{=} L_{0}^{2}(\Omega)$ as the fluid velocity and pressure functional spaces, respectively. The space of admissible displacements is denoted by $\boldsymbol{Y} \subset\left[H^{1}(\Sigma)\right]^{2}$. The trial set for the unitary director vectors is denoted by $\boldsymbol{S}$ and its variations are taken in the space $\Theta \subset H^{1}(\Sigma)$.

\subsection{Fitted mesh methods}

By using the parametrization of the fluid domain, given by the one-to-one mapping $\mathcal{A}$, we can consider as fluid unknowns

$$
\widehat{\boldsymbol{u}} \stackrel{\text { def }}{=} \boldsymbol{u} \circ \mathcal{A}_{t}, \quad \widehat{p} \stackrel{\text { def }}{=} p \circ \mathcal{A}_{t},
$$

instead of $\boldsymbol{u}=\widehat{\boldsymbol{u}} \circ \mathcal{A}_{t}^{-1}$ and $p=\widehat{p} \circ \mathcal{A}_{t}^{-1}$. The coupled problem (5)-(7) hence admits the following variational formulation (see, e.g., [24]): for $t>0$, find $(\widehat{\boldsymbol{u}}, \widehat{p}, \boldsymbol{d}, \boldsymbol{a}) \in \boldsymbol{V} \times Q \times \boldsymbol{Y} \times \boldsymbol{S}$, with $\dot{\boldsymbol{d}}=\partial_{t} \boldsymbol{d}$ and $\left.\widehat{\boldsymbol{u}}\right|_{\Sigma}=\dot{\boldsymbol{d}}$, such that the geometric compatibility $(7)_{1}$ holds and

$$
\left\{\begin{array}{l}
\rho^{\mathrm{f}} \frac{\mathrm{d}}{\mathrm{d} t}(\boldsymbol{u}, \boldsymbol{v})_{\Omega(t)}-\rho^{\mathrm{f}}((\operatorname{div} \boldsymbol{w}) \boldsymbol{u}, \boldsymbol{v})_{\Omega(t)}+a_{\Omega(t)}^{\mathrm{f}}(\boldsymbol{u}-\boldsymbol{w} ;(\boldsymbol{u}, p),(\boldsymbol{v}, q)) \\
+\rho^{\mathrm{s}} \epsilon\left(\partial_{\tau} \dot{\boldsymbol{d}}, \boldsymbol{y}\right)_{\Sigma}+a^{\mathrm{s}}((\boldsymbol{d}, \boldsymbol{a}),(\boldsymbol{y}, \theta))=0
\end{array}\right.
$$

for all $(\widehat{\boldsymbol{v}}, \widehat{q}, \boldsymbol{y}, \theta) \in \boldsymbol{V} \times Q \times \boldsymbol{Y} \times \Theta$ with $\left.\widehat{\boldsymbol{v}}\right|_{\Sigma}=\boldsymbol{y}$. Here, the trilinear fluid form is defined by

$$
a_{\Omega(t)}^{\mathrm{f}}(\boldsymbol{z} ;(\boldsymbol{u}, p),(\boldsymbol{v}, q)) \stackrel{\text { def }}{=} c_{\Omega(t)}(\boldsymbol{z}, \boldsymbol{u}, \boldsymbol{v})+a_{\Omega(t)}((\boldsymbol{u}, p),(\boldsymbol{v}, q)),
$$

with

$$
\begin{aligned}
& a_{\Omega(t)}((\boldsymbol{u}, p),(\boldsymbol{v}, q)) \stackrel{\text { def }}{=} 2 \mu(\boldsymbol{\epsilon}(\boldsymbol{u}), \boldsymbol{\epsilon}(\boldsymbol{v}))_{\Omega(t)}-(p, \operatorname{div} \boldsymbol{v})_{\Omega(t)}+(q, \operatorname{div} \boldsymbol{u})_{\Omega(t)}, \\
& c_{\Omega(t)}(\boldsymbol{z}, \boldsymbol{u}, \boldsymbol{v}) \stackrel{\text { def }}{=} \rho^{\mathrm{f}}(\boldsymbol{z} \cdot \boldsymbol{\nabla} \boldsymbol{u}, \boldsymbol{v})_{\Omega(t)} .
\end{aligned}
$$

The term $a^{\mathrm{s}}$ represents the weak form of the solid operators $\boldsymbol{L}_{\boldsymbol{d}}$ and $\boldsymbol{L}_{\boldsymbol{a}}$.

The coupled variational problem $(8)$ is particularly well adapted to the discretization with fitted meshes. We hence consider a family of triangulations of $\left\{\mathcal{T}_{h}^{\mathrm{f}}\right\}_{0<h<1}$ of the reference fluid domain $\Omega$ which are fitted to the family of triangulations $\left\{\mathcal{T}_{h}^{\mathrm{s}}\right\}_{0<h<1}$ of $\Sigma$. In other words, the fluid and solid meshes match at the interface. The discrete spaces $\boldsymbol{V}_{h} \subset \boldsymbol{V}, \boldsymbol{Y}_{h} \subset \boldsymbol{Y}$ and $\Theta_{h} \subset \Theta$ are the standard Lagrange finite element space of continuous piece-wise affine functions, so that we have $\boldsymbol{Y}_{h}=\left.\boldsymbol{V}_{h}\right|_{\Sigma}$. The pressure space $Q_{h} \subset Q$ is also made of piece-wise affine functions which are continuous on each sub-domain $\Omega_{i}, i=1,2$, but which can be discontinuous across $\Sigma$.

The geometric coupling condition $(7)_{1}$ is treated in an explicit fashion as follows. For a given discrete displacement $\boldsymbol{d}_{h}^{n-1} \in \boldsymbol{Y}_{h}$ at time level $n-1$, we define the ALE map $\mathcal{A}_{h}^{n}$ as

$$
\boldsymbol{d}_{h}^{\mathrm{f}, n}=\mathcal{L}_{h}\left(\boldsymbol{d}_{h}^{n-1}\right), \quad \mathcal{A}_{h}^{n}=\boldsymbol{I}_{\Omega}+\boldsymbol{d}_{h}^{\mathrm{f}, n} .
$$


Algorithm 1 Fitted mesh ALE method.

For $n \geq 1$,

1. Fluid mesh update:

$$
\boldsymbol{d}_{h}^{\mathrm{f}, n}=\mathcal{L}_{h}\left(\boldsymbol{d}_{h}^{n-1}\right), \quad \boldsymbol{w}_{h}^{n}=\partial_{\tau} \boldsymbol{d}_{h}^{\mathrm{f}, n}, \quad \mathcal{A}_{h}^{n}=\boldsymbol{I}_{\Omega}+\boldsymbol{d}_{h}^{\mathrm{f}, n}, \quad \Omega_{h}^{n}=\mathcal{A}_{h}^{n}(\Omega) .
$$

2. Find $\left(\widehat{\boldsymbol{u}}_{h}^{n}, \widehat{p}_{h}^{n}, \boldsymbol{d}_{h}^{n}, \boldsymbol{a}_{h}^{n}\right) \in \boldsymbol{V}_{h} \times Q_{h} \times \boldsymbol{Y}_{h} \times \boldsymbol{S}_{h}$, with $\dot{\boldsymbol{d}}_{h}=\partial_{\tau} \boldsymbol{d}_{h}^{n}$ and $\left.\widehat{\boldsymbol{u}}_{h}^{n}\right|_{\Sigma}=\dot{\boldsymbol{d}}_{h}^{n}$, such that

$$
\left\{\begin{array}{l}
\frac{\rho^{\mathrm{f}}}{\tau}\left[\left(\boldsymbol{u}_{h}^{n}, \boldsymbol{v}_{h}\right)_{\Omega_{h}^{n}}-\left(\boldsymbol{u}_{h}^{n-1}, \boldsymbol{v}_{h}\right)_{\Omega_{h}^{n-1}}\right]-\rho^{\mathrm{f}}\left(\left(\operatorname{div} \boldsymbol{w}_{h}^{n}\right) \boldsymbol{u}_{h}^{n}, \boldsymbol{v}_{h}\right)_{\Omega_{h}^{n}} \\
+a_{\Omega_{h}^{n}, h}^{\mathrm{f}}\left(\boldsymbol{u}_{h}^{n-1}-\boldsymbol{w}_{h}^{n} ;\left(\boldsymbol{u}_{h}^{n}, p_{h}^{n}\right),\left(\boldsymbol{v}_{h}, q_{h}\right)\right) \\
+\rho^{\mathrm{s}} \epsilon\left(\partial_{\tau} \dot{\boldsymbol{d}}_{h}^{n}, \boldsymbol{y}_{h}\right)_{\Sigma}+a_{h}^{\mathrm{s}}\left(\left(\boldsymbol{d}_{h}^{n}, \boldsymbol{a}_{h}^{n}\right),\left(\boldsymbol{y}_{h}, \theta_{h}\right)\right)=0,
\end{array}\right.
$$

for all $\left(\widehat{\boldsymbol{v}}_{h}, \widehat{q}_{h}, \boldsymbol{y}_{h}, \theta_{h}\right) \in \boldsymbol{V}_{h} \times Q_{h} \times \boldsymbol{Y}_{h} \times \Theta_{h}$ with $\left.\boldsymbol{v}_{h}\right|_{\Sigma}=\boldsymbol{y}_{h}$.

The resulting numerical method is detailled in Algorithm 1. Here, we have used the standard notation

$$
\begin{aligned}
a_{\Omega_{h}^{n}, h}^{\mathrm{f}}\left(\boldsymbol{z}_{h} ;\left(\boldsymbol{u}_{h}, p_{h}\right),\left(\boldsymbol{v}_{h}, q_{h}\right)\right) \stackrel{\text { def }}{=} & c_{h}^{n}\left(\boldsymbol{z}_{h} ; \boldsymbol{u}_{h}, \boldsymbol{v}_{h}\right)+a_{\Omega_{h}^{n}}\left(\left(\boldsymbol{u}_{h}, p_{h}\right),\left(\boldsymbol{v}_{h}, q_{h}\right)\right) \\
& +s_{\Omega_{h}^{n}, h}\left(\boldsymbol{z}_{h} ; \boldsymbol{u}_{h}, \boldsymbol{v}_{h}\right),
\end{aligned}
$$

with the convective trilinear form given by

$$
c_{h}^{n}\left(\boldsymbol{z}_{h} ; \boldsymbol{u}_{h}, \boldsymbol{v}_{h}\right) \stackrel{\text { def }}{=} c_{\Omega_{h}^{n}}\left(\boldsymbol{z}_{h} ; \boldsymbol{u}_{h}, \boldsymbol{v}_{h}\right)+\frac{\rho^{\mathrm{f}}}{2}\left(\left(\operatorname{div} \boldsymbol{z}_{h}\right) \boldsymbol{u}_{h}, \boldsymbol{v}_{h}\right)_{\Omega_{h}^{n}}
$$

and the form $s_{\Omega_{h}^{n}, h}$ corresponds to the SUPG/PSPG and grad-div stabilizations given by (see, e.g., [47]):

$$
\begin{aligned}
& s_{\Omega_{h}^{n}, h}\left(\boldsymbol{z}_{h} ; \boldsymbol{u}_{h}, \boldsymbol{v}_{h}\right) \stackrel{\text { def }}{=} \sum_{K \in \mathcal{T}_{h}^{\mathrm{f}}} \int_{K} \frac{\lambda_{\mathrm{C}} h^{2}}{\delta_{h}} \operatorname{div} \boldsymbol{u}_{h} \operatorname{div} \boldsymbol{v}_{h} \\
& \quad+\sum_{K \in \mathcal{T}_{h}^{\mathrm{f}}} \int_{K} \delta_{h}\left(\rho^{\mathrm{f}}\left(\boldsymbol{z}_{h} \cdot \nabla\right) \boldsymbol{u}_{h}+\nabla p_{h}\right) \cdot\left(\rho^{\mathrm{f}}\left(\boldsymbol{z}_{h} \cdot \nabla\right) \boldsymbol{v}_{h}+\nabla q_{h}\right), \\
& \delta_{h} \stackrel{\text { def }}{=} \lambda_{\mathrm{M}}\left(\rho^{\mathrm{f}} \sqrt{\frac{4}{\tau^{2}}+\frac{16 \mu^{2}}{h^{4}\left(\rho^{\mathrm{f}}\right)^{2}}+\frac{4\left|\boldsymbol{z}_{h}\right|^{2}}{h^{2}}}\right)^{-1},
\end{aligned}
$$

with $\lambda_{\mathrm{M}}, \lambda_{\mathrm{C}}>0$ user-defined parameters. At last, the solid term $a_{h}^{\mathrm{s}}$ denotes the linear MITC (Mixed Interpolation of Tensorial Components approach) approximation of $a^{\mathrm{s}}$ (see, e.g., 6]), involving 3 degrees of freedom per node in the increments (two displacements and one rotation).

Remark 3.1. In (9), the fluid integrals in the current configurations involve the composition with the corresponding discrete ALE map. For instance, the second term is given by

$$
\left(\boldsymbol{u}_{h}^{n-1}, \boldsymbol{v}_{h}\right)_{\Omega_{h}^{n}}=\left(\widehat{\boldsymbol{u}}_{h}^{n-1} \circ\left(\mathcal{A}_{h}^{n}\right)^{-1}, \widehat{\boldsymbol{v}}_{h} \circ\left(\mathcal{A}_{h}^{n}\right)^{-1}\right)_{\Omega_{h}^{n}} .
$$

The main drawback of Algorithm 1 comes from the motion of the fluid domain mesh in step 1. Indeed, the viability and efficiency of the whole numerical method strongly depends on 
the capability of the discrete lifting operator $\mathcal{L}_{h}$ to deliver a correct deformed fluid mesh which maintains a reasonable quality. This contraint often becomes a challenging problem in the case of large interface deflections (see, e.g., 46, 51, 1]). In this paper, we consider the non-linear lifting operator introduced in 36 which is simply an incremental variant of the approach reported in [46].

\subsection{Unfitted mesh methods}

The coupled problem (1)-3) is often discretized with an unfitted mesh method to circumvent the above mentioned limitation of Algorithm 1 . In this paper, we consider two different unfitted methods which are discussed in the next two paragraphs.

\subsubsection{Fictitious domain method}

The basic idea of the so-called Fictitious Domain method, is to consider the kinematic condition $(3)_{2}$ as a constraint in the fluid problem which is enforced through Lagrange multipliers, viz.,

$$
b(\boldsymbol{\mu}, \boldsymbol{u} \circ \boldsymbol{\phi})=b(\boldsymbol{\mu}, \dot{\boldsymbol{d}}), \quad \forall \boldsymbol{\mu} \in \boldsymbol{\Lambda},
$$

where $\boldsymbol{\Lambda}$ denotes the Lagrange multiplier space and $b: \boldsymbol{\Lambda} \times\left[H^{\frac{1}{2}}(\Sigma)\right]^{2} \rightarrow \mathbb{R}$ is a continuous bilinear form, such that $b(\boldsymbol{\mu}, \boldsymbol{z})=0$ for all $\boldsymbol{\mu} \in \boldsymbol{\Lambda}$ yields $\boldsymbol{z}=\mathbf{0}$ on $\Sigma$. For instance, we can take $\boldsymbol{\Lambda}=\left(\left[H^{\frac{1}{2}}(\Sigma)\right]^{2}\right)^{\prime}$ and $b(\boldsymbol{\mu}, \boldsymbol{z})=\langle\boldsymbol{\mu}, \boldsymbol{z}\rangle$, where $\langle\cdot, \cdot\rangle$ represents the duality pairing between $\left(\left[H^{\frac{1}{2}}(\Sigma)\right]^{2}\right)^{\prime}$ and $\left[H^{\frac{1}{2}}(\Sigma)\right]^{2}$ (see, e.g., [39, 11]).

Hence, in weak form the coupled problem (1)-(3) can be formulated as follows: for $t>0$, find $(\boldsymbol{u}, p, \boldsymbol{\lambda}, \boldsymbol{d}, \boldsymbol{a}) \in \boldsymbol{V} \times Q \times \boldsymbol{\Lambda} \times \boldsymbol{Y} \times \boldsymbol{S}$, with $\boldsymbol{d}=\partial_{t} \boldsymbol{d}$ such that the geometric compatibility (3) 1 holds and

$$
\left\{\begin{array}{l}
\rho^{\mathrm{f}}\left(\partial_{t} \boldsymbol{u}, \boldsymbol{v}\right)_{\Omega}+a_{\Omega}^{\mathrm{f}}(\boldsymbol{u} ;(\boldsymbol{u}, p),(\boldsymbol{v}, q))+\rho^{\mathrm{s}} \epsilon\left(\partial_{t} \dot{\boldsymbol{d}}, \boldsymbol{y}\right)_{\Sigma}+a^{\mathrm{s}}((\boldsymbol{d}, \boldsymbol{a}),(\boldsymbol{y}, \theta)) \\
+b\left(\boldsymbol{\lambda}, \boldsymbol{v} \circ \boldsymbol{\phi}_{t}-\boldsymbol{y}\right)-b\left(\boldsymbol{\mu}, \boldsymbol{u} \circ \boldsymbol{\phi}_{t}-\dot{\boldsymbol{d}}\right)=0,
\end{array}\right.
$$

for all $(\boldsymbol{v}, q, \boldsymbol{\mu}, \boldsymbol{y}, \theta) \in \boldsymbol{V} \times Q \times \boldsymbol{\Lambda} \times \boldsymbol{Y} \times \Theta$.

Algorithm 2 Fictitious domain method (from [21]).

For $n \geq 1$,

1. Interface update:

$$
\phi_{h}^{n}=\boldsymbol{I}_{\Sigma}+\boldsymbol{d}_{h}^{n-1}, \quad \Sigma_{h}^{n}=\boldsymbol{\phi}_{h}^{n}(\Sigma), \quad \Omega_{h}^{n}=\Omega \backslash \Sigma_{h}^{n} .
$$

2. Find $\left(\boldsymbol{u}_{h}^{n}, p_{h}^{n}, \boldsymbol{\lambda}_{h}^{n}, \boldsymbol{d}_{h}^{n}, \boldsymbol{a}_{h}^{n}\right) \in \boldsymbol{V}_{h} \times Q_{h} \times \boldsymbol{\Lambda}_{h} \times \boldsymbol{Y}_{h} \times \boldsymbol{S}_{h}$, with $\dot{\boldsymbol{d}}_{h}=\partial_{\tau} \boldsymbol{d}_{h}^{n}$, such that

$$
\left\{\begin{array}{l}
\rho^{\mathrm{f}}\left(\partial_{\tau} \boldsymbol{u}_{h}^{n}, \boldsymbol{v}_{h}\right)_{\Omega}+a_{\Omega, h}^{\mathrm{f}}\left(\boldsymbol{u}_{h}^{n-1} ;\left(\boldsymbol{u}_{h}^{n}, p_{h}^{n}\right),\left(\boldsymbol{v}_{h}, q_{h}\right)\right) \\
+\rho^{\mathrm{s}} \epsilon\left(\partial_{\tau} \dot{\boldsymbol{d}}_{h}^{n}, \boldsymbol{y}_{h}\right)_{\Sigma}+a^{\mathrm{s}}\left(\left(\boldsymbol{d}_{h}^{n}, \boldsymbol{a}_{h}^{n}\right),\left(\boldsymbol{y}_{h}, \theta_{h}\right)\right) \\
+b\left(\boldsymbol{\lambda}_{h}^{n}, \boldsymbol{v}_{h} \circ \boldsymbol{\phi}_{h}^{n}-\boldsymbol{w}_{h}\right)-b\left(\boldsymbol{\mu}_{h}, \boldsymbol{u}_{h}^{n} \circ \boldsymbol{\phi}_{h}^{n}-\dot{\boldsymbol{d}}_{h}^{n}\right)=0,
\end{array}\right.
$$

for all $\left(\boldsymbol{v}_{h}, q_{h}, \boldsymbol{\mu}_{h}, \boldsymbol{y}_{h}, \theta_{h}\right) \in \boldsymbol{V}_{h} \times Q_{h} \times \boldsymbol{\Lambda}_{h} \times \boldsymbol{Y}_{h} \times \Theta_{h}$.

At the discrete level, the fluid velocity is searched for in the same space $\boldsymbol{V}_{h}$ as in the fitted mesh formulation of Section 3.1. Nevertheless, the fluid mesh is now fixed and the pressure space 
$Q_{h}$ is made of globally continuous functions. For the solid, we consider the same discretization as in Section 3.1. As approximation space for the Lagrange multipliers, we take

$$
\boldsymbol{\Lambda}_{h}=\left\{\boldsymbol{\mu}_{h}=\sum_{i=1}^{N_{h}^{\mathrm{s}}} \boldsymbol{\mu}_{i} \delta_{\boldsymbol{x}_{i}^{\mathrm{s}}} / \boldsymbol{\mu}_{i} \in \mathbb{R}^{2}, \quad i=1, \ldots, N_{h}^{\mathrm{s}}\right\},
$$

where $\left\{\boldsymbol{x}_{i}^{\mathrm{s}}\right\}_{i=1}^{N_{h}^{\mathrm{s}}}$ denotes the points of the triangulation $\mathcal{T}_{h}^{\mathrm{s}}$ and $\delta_{\boldsymbol{x}_{i}^{\mathrm{s}}}$ stands for the Dirac's measure at point $\boldsymbol{x}_{i}^{\mathrm{s}}$. This non-conforming approximation was used for example in 28, 21] and analyzed in [23. The discrete method is hence obtained from the approximation of the weak form [11], as detailed in Algorithm2. Note that the geometric compatibility condition $[3)_{1}$ is treated explicitly via step 1.

From (13), the discrete treatment of the kinematic constraint in 12 , namely,

$$
b\left(\boldsymbol{\mu}_{h}, \boldsymbol{u}_{h}^{n} \circ \phi_{h}^{n}-\dot{\boldsymbol{d}}_{h}^{n}\right)=0 \quad \forall \boldsymbol{u}_{h} \in \boldsymbol{\Lambda}_{h},
$$

simply reads:

$$
\boldsymbol{u}_{h} \circ \phi_{h}\left(\boldsymbol{x}_{i}^{\mathrm{s}}\right)-\dot{\boldsymbol{d}}_{h}\left(\boldsymbol{x}_{i}^{\mathrm{s}}\right)=0, \quad \forall i=1, \ldots, N_{h}^{\mathrm{s}} .
$$

The present computer implementation of this method is performed by penalization (omitted in the concise description of Algorithm 2 for the sake of clarity). Let $\varepsilon>0$ be a given (nondimensionless) parameter, the kinematic coupling condition (14) is hence replaced by:

$$
\boldsymbol{u}_{h} \circ \phi_{h}\left(\boldsymbol{x}_{i}^{\mathrm{s}}\right)-\dot{\boldsymbol{d}}_{h}\left(\boldsymbol{x}_{i}^{\mathrm{s}}\right)=\varepsilon \lambda_{i}, \quad \forall i=1, \ldots, N_{h}^{\mathrm{s}} .
$$

Owing to the choice of $\boldsymbol{\Lambda}_{h}$, the elimination of the Lagrange multipliers obtained with the penalization can be done without affecting the sparse pattern of the matrix of the fluid problem. This property makes the approach very convenient from a computer implementation point of view. The influence of the penalty parameter $\varepsilon$ on the accuracy of the method will be discussed in Section 4, devoted to the numerical experiments. The choice here is to enforce relation (15) at every time step, but let us mention an interesting alternative which consists in introducing a time dynamics for the Lagrange multiplier, in the spirit of what is done for the pressure in pseudo-compressibility methods. This approach is adopted in 34, and further analyzed in 35] in a scalar case.

The main sources of inaccuracies in Algorithm 2 come from the choice of the Lagrange multiplier space $(13)$ and from the continuous nature of the pressure approximation across the interface. While the impact of the former can be mitigated via mesh refinement, the latter is known to yield major mass conservation issues across the interface. It is worth noting that similar poor mass conservation was encountered with the original immersed boundary method using finite difference approximations in space (see, e.g., [41, [5]). In the variational setting of Algorithm 2 the use of alternative spatial approximations, based on globally discontinuous pressures and higher order polynomials for the velocities, has been investigated in [4, 8, 7, 10] with the purpose of overcoming this issue. In this paper, we follow the approach considered in 34. (see also [25, 15), with which the original (low-order) velocity/pressure approximation is preserved by simply modifying the SUPG/PSPG and grad-div stabilizations in a neighborhood $\omega_{h}^{n}$ of the interface $\Sigma_{h}^{n}$ (typically 2 layers of fluid elements in each side). More precisely, in 10 we take

$$
\lambda_{\mathrm{C}}=1 \quad \text { in } \quad \Omega, \quad \lambda_{\mathrm{M}}=\left\{\begin{array}{rll}
1 & \text { in } & \Omega \backslash \omega_{h}^{n}, \\
\varepsilon_{\mathrm{M}} & \text { in } & \omega_{h}^{n},
\end{array}\right.
$$

with $0<\varepsilon_{\mathrm{M}} \ll 1$ a user-defined parameter. Thus, the idea of the method consists in boosting the grad-div stabilization while reducing the SUPG/PSPG stabilization near the interface. 
The first improves mass conservation while the second limits the impact of the local residual inconsistencies.

\subsubsection{Nitsche-XFEM method}

As in Algorithm 2, the geometric compatibility condition $(3)_{1}$ is treated in an explicit fashion and we consider the same discretization of the solid. For the construction of the fluid approximation spaces, we introduce two family of meshes $\left\{\mathcal{T}_{h, i}^{n}\right\}_{0<h<1}, i=1,2$, where each $\mathcal{T}_{h, i}^{n}$ covers the $i$-th fluid region defined by $\Sigma_{h}^{n}$. Each mesh $\mathcal{T}_{h, i}^{n}$ is fitted to the exterior boundary $\Gamma$ but not to $\Sigma_{h}^{n}$ (see Figure 3). Furthermore for every element $K \in \mathcal{T}_{h, 1}^{n} \cap \mathcal{T}_{h, 2}^{n}$ we assume that $K \cap \Sigma_{h}^{n} \neq \emptyset$. Note

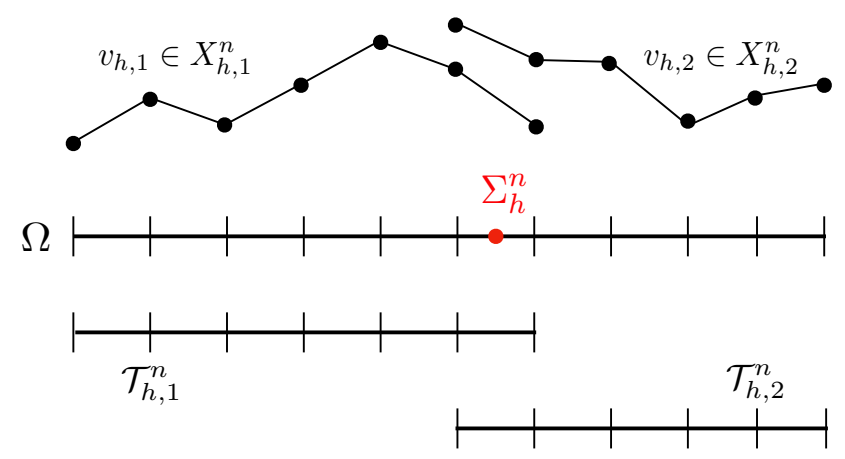

Figure 3: One dimensional illustration of the construction of the discrete spaces $X_{h, i}^{n}$.

that $\mathcal{T}_{h, 1}^{n} \cup \mathcal{T}_{h, 2}^{n}$ is a triangulation of the whole fluid domain $\Omega$. We denote by $\Omega_{h, i}^{n}$ the domain covered by $\mathcal{T}_{h, i}^{n}$, viz.,

$$
\Omega_{h, i}^{n} \stackrel{\text { def }}{=} \operatorname{int}\left(\cup_{K \in \mathcal{T}_{h, i}^{n}} K\right) .
$$

We can hence introduce the following time level dependent spaces of continuous piecewise affine functions:

$$
X_{h, i}^{n} \stackrel{\text { def }}{=}\left\{v_{h} \in C^{0}\left(\overline{\Omega_{h, i}^{n}}\right) / v_{h \mid K} \in \mathbb{P}_{1}(K), \quad \forall K \in \mathcal{T}_{h, i}^{n}\right\},
$$

for $i=1,2$. For the approximation of the fluid velocity and pressure we will consider the product spaces

$$
\boldsymbol{V}_{h}^{n} \stackrel{\text { def }}{=}\left(\left[X_{h, 1}^{n}\right]^{2} \times\left[X_{h, 2}^{n}\right]^{2}\right) \cap \boldsymbol{V}, \quad Q_{h}^{n} \stackrel{\text { def }}{=}\left(X_{h, 1}^{n} \times X_{h, 2}^{n}\right) \cap Q,
$$

respectively. Note that interfacial (strong and weak) discontinuities are now incorporated at the discrete level. Indeed, the functions of these spaces are continuous in the fluid domain $\Omega_{h}^{n} \stackrel{\text { def }}{=} \Omega \backslash \Sigma_{h}^{n}$, but discontinuous across the moving interface $\Sigma_{h}^{n}$ (see Figure 3 ).

In this framework, the kinematic/dynamic fluid-solid coupling $(3)_{2,3}$ can be enforced in a consistent fashion (and Lagrange multipliers free) through a fluid-sided Nitsche's mortaring (see [2, 13]). The resulting numerical method is detailed in Algorithm 3

Here, $\gamma>0$ denotes the Nitsche's penalty parameter and the fluid trilinear form $a_{h}^{\mathrm{f}, n}$ is given by the following expression

$$
\begin{aligned}
a_{h}^{\mathrm{f}, n}\left(\boldsymbol{z}_{h} ;\left(\boldsymbol{u}_{h}, p_{h}\right),\left(\boldsymbol{v}_{h}, q_{h}\right)\right) \stackrel{\text { def }}{=} c_{h}^{n}\left(\boldsymbol{z}_{h} ; \boldsymbol{u}_{h}, \boldsymbol{v}_{h}\right)+a_{\Omega_{h}^{n}}\left(\left(\boldsymbol{u}_{h}, p_{h}\right),\left(\boldsymbol{v}_{h}, q_{h}\right)\right) \\
\\
+s_{\mathrm{v}, h}^{n}\left(\boldsymbol{z}_{h} ; \boldsymbol{u}_{h}, \boldsymbol{v}_{h}\right)+s_{\mathrm{p}, h}^{n}\left(\boldsymbol{z}_{h} ; p_{h}, q_{h}\right)+g_{h}^{n}\left(\boldsymbol{u}_{h}, \boldsymbol{v}_{h}\right) .
\end{aligned}
$$


Algorithm 3 Nitsche-XFEM method (from [2]).

For $n \geq 1$,

1. Interface update:

$$
\boldsymbol{\phi}_{h}^{n}=\boldsymbol{I}_{\Sigma}+\boldsymbol{d}_{h}^{n-1}, \quad \Sigma_{h}^{n}=\phi_{h}^{n}(\Sigma), \quad \Omega_{h}^{n}=\Omega \backslash \Sigma_{h}^{n} .
$$

2. Find $\left(\boldsymbol{u}_{h}^{n}, p_{h}^{n}, \boldsymbol{d}_{h}^{n}, \boldsymbol{a}_{h}^{n}\right) \in \boldsymbol{V}_{h}^{n} \times Q_{h}^{n} \times \boldsymbol{Y}_{h} \times \boldsymbol{S}_{h}$, with $\dot{\boldsymbol{d}}_{h}=\partial_{\tau} \boldsymbol{d}_{h}^{n}$ and such that

$$
\left\{\begin{array}{l}
\rho^{\mathrm{f}}\left(\partial_{\tau} \boldsymbol{u}_{h}^{n}, \boldsymbol{v}_{h}\right)_{\Omega_{h}^{n}}+a_{h}^{\mathrm{f}, n}\left(\boldsymbol{u}_{h}^{n-1} ;\left(\boldsymbol{u}_{h}^{n}, p_{h}^{n}\right),\left(\boldsymbol{v}_{h}, q_{h}\right)\right) \\
+\rho^{\mathrm{s}} \epsilon\left(\partial_{\tau} \dot{\boldsymbol{d}}_{h}^{n}, \boldsymbol{y}_{h}\right)_{\Sigma}+a_{h}^{\mathrm{s}}\left(\left(\boldsymbol{d}_{h}^{n}, \boldsymbol{a}_{h}^{n}\right),\left(\boldsymbol{y}_{h}, \theta_{h}\right)\right) \\
-\sum_{i=1}^{2}\left(\boldsymbol{\sigma}\left(\boldsymbol{u}_{h, i}^{n}, p_{h, i}^{n}\right) \boldsymbol{n}_{i}, \boldsymbol{v}_{h, i}-\boldsymbol{y}_{h}\right)_{\Sigma_{h}^{n}} \\
-\sum_{i=1}^{2}\left(\boldsymbol{u}_{h, i}^{n}-\dot{\boldsymbol{d}}_{h}^{n}, \boldsymbol{\sigma}\left(\boldsymbol{v}_{h, i},-q_{h, i}\right) \boldsymbol{n}_{i}\right)_{\Sigma_{h}^{n}} \\
+\frac{\gamma \mu}{h} \sum_{i=1}^{2}\left(\boldsymbol{u}_{h, i}^{n}-\dot{\boldsymbol{d}}_{h}^{n}, \boldsymbol{v}_{h, i}-\boldsymbol{y}_{h}\right)_{\Sigma_{h}^{n}}=0
\end{array}\right.
$$

for all $\left(\boldsymbol{v}_{h}, q_{h}, \boldsymbol{y}_{h}, \theta_{h}\right) \in \boldsymbol{V}_{h}^{n} \times Q_{h}^{n} \times \boldsymbol{Y}_{h} \times \Theta_{h}$.

In this expression, the convective term is defined as

$$
\begin{aligned}
c_{h}^{n}\left(\boldsymbol{z}_{h} ; \boldsymbol{u}_{h}, \boldsymbol{v}_{h}\right) \stackrel{\text { def }}{=} c_{\Omega_{h}^{n}}\left(\boldsymbol{z}_{h} ; \boldsymbol{u}_{h}, \boldsymbol{v}_{h}\right) & +\frac{\rho^{\mathrm{f}}}{2}\left(\left(\operatorname{div} \boldsymbol{z}_{h}\right) \boldsymbol{u}_{h}, \boldsymbol{v}_{h}\right)_{\Omega_{h}^{n}} \\
& -\rho^{\mathrm{f}}\left(\left\{\left\{\boldsymbol{z}_{h}\right\} \cdot \boldsymbol{n} \llbracket \boldsymbol{u}_{h} \rrbracket,\left\{\left\{\boldsymbol{v}_{h} \rrbracket\right)_{\Sigma_{h}^{n}}-\frac{\rho^{\mathrm{f}}}{2}\left(\llbracket \boldsymbol{z}_{h} \cdot \boldsymbol{n} \rrbracket,\left\{\boldsymbol{u}_{h} \cdot \boldsymbol{v}_{h} \rrbracket\right)_{\Sigma_{h}^{n}},\right.\right.\right.\right.
\end{aligned}
$$

where the last two (consistent) terms are introduced for stability purposes to guarantee that $c_{h}^{n}\left(\boldsymbol{z}_{h} ; \boldsymbol{v}_{h}, \boldsymbol{v}_{h}\right)=0$.

The terms in $s_{\mathrm{v}, h}^{n}$ and $s_{\mathrm{p}, h}^{n}$ in 17 correspond to the continuous interior penalty (CIP) stabilization (see [14]) given by:

$$
\begin{aligned}
& s_{\mathrm{v}, h}^{n}\left(\boldsymbol{z}_{h} ; \boldsymbol{u}_{h}, \boldsymbol{v}_{h}\right) \stackrel{\text { def }}{=} \gamma_{\mathrm{v}} h^{2} \sum_{i=1}^{2} \sum_{F \in \mathcal{F}_{h, i}^{n}} \xi_{F}\left(\boldsymbol{z}_{h}\right)\left\|\boldsymbol{z}_{h} \cdot \boldsymbol{n}\right\|_{L^{\infty}(F)}\left(\llbracket \boldsymbol{\nabla} \boldsymbol{u}_{h} \rrbracket_{F}, \llbracket \boldsymbol{\nabla} \boldsymbol{v}_{h} \rrbracket_{F}\right)_{F}, \\
& s_{\mathrm{p}, h}^{n}\left(\boldsymbol{z}_{h} ; p_{h}, q_{h}\right) \stackrel{\text { def }}{=} \gamma_{\mathrm{p}} h^{2} \sum_{i=1}^{2} \sum_{F \in \mathcal{F}_{h, i}^{n}} \frac{\xi_{F}\left(\boldsymbol{z}_{h}\right)}{\left\|\boldsymbol{z}_{h}\right\|_{L^{\infty}(F)}}\left(\llbracket \nabla p_{h} \rrbracket_{F}, \llbracket \nabla q_{h} \rrbracket_{F}\right)_{F},
\end{aligned}
$$

where $\mathcal{F}_{h, i}^{n}$ denotes the set of interior edges of $\mathcal{T}_{h, i}^{n}$, the symbol $\llbracket \cdot \rrbracket_{F}$ stands for the jump operator across the edge $F$,

$$
\xi_{F}\left(\boldsymbol{z}_{h}\right) \stackrel{\text { def }}{=} \min \left\{1, \operatorname{Re}_{F}\left(\boldsymbol{z}_{h}\right)\right\}, \quad \operatorname{Re}_{F}\left(\boldsymbol{z}_{h}\right) \stackrel{\text { def }}{=} \frac{\rho^{\mathrm{f}}\left\|\boldsymbol{z}_{h}\right\|_{L^{\infty}(F)} h}{\mu},
$$

and $\gamma_{\mathrm{p}}, \gamma_{\mathrm{v}}>0$ are user-defined parameters. The main reason of using the CIP stabilization (instead of $(10)$ ) is that it is strongly consistent in the whole computational domain $\Omega_{h, 1}^{n} \times \Omega_{h, 2}^{n}$, 
while (10) it is strongly consistent only in the physical domain $\Omega_{h}^{n}$ due to its residual-based nature (see [2, Section 3.2.1] for a discussion).

At last, the term $g_{h}^{n}$ in 17 corresponds to the so-called ghost-penalty stabilization operator which guarantees the robustness of the method with respect to the way the interface $\Sigma_{h}^{n}$ cuts the fluid elements. It is given by the following expression (see [12]):

$$
g_{h}^{n}\left(\boldsymbol{u}_{h}, \boldsymbol{v}_{h}\right) \stackrel{\text { def }}{=} \gamma_{\mathrm{g}} \mu h \sum_{i=1}^{2} \sum_{F \in \mathcal{F}_{h, i}^{n, \Sigma}}\left(\llbracket \nabla \boldsymbol{u}_{h, i} \rrbracket_{F}, \llbracket \nabla \boldsymbol{v}_{h, i} \rrbracket_{F}\right)_{F}
$$

where $\mathcal{F}_{h, i}^{n, \Sigma}$ denotes the set of interior edges of the elements of $\mathcal{T}_{h, i}^{n}$ intersected by $\Sigma_{h}^{n}$ and $\gamma_{\mathrm{g}}>0$ is a user-defined parameter.

It should be noted that, for consistency, all the fluid bulk terms in (16) are evaluated only in the physical zones of $\Omega_{h, i}^{n}$ (i.e., the integrals are evaluated over cut-elements). Furthermore, the interface integrals on $\Sigma_{h}^{n}$ involve the products of fields defined on different meshes. Hence, in comparison with Algorithm 2, Algorithm 3 requires a non-standard computer implementation and a specific evaluation of all the interface intersections. We refer to [2, Section 2.1.4] (and the references therein) for a critical review on this point.

\section{Numerical experiments}

The purpose of this section is to illustrate and compare, via a series of 2D-numerical examples, the accuracy of the unfitted methods described in Section 3.2. To this purpose, we consider as reference solution the one provided by the fitted mesh method (Algorithm 1) using a highly refined space-time grid. The accuracy of the unfitted mesh methods will be then evaluated by comparing the results obtained with three successive levels of mesh refinement in space and in time.

Through this section, the acronyms ALE, FD and NXFEM respectively refer to Algorithms 1,2 and 3. Moreover, the variant of Algorithm 2 discussed in Section 3.2.1, with enhanced interfacial

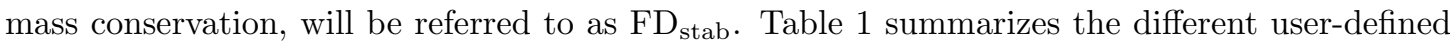
parameters involved in each method, with some prescribed values. For the FD and FD stab meth-

\begin{tabular}{|c|c|c|c|}
\hline$\overline{\mathrm{ALE}}$ & FD & $\mathrm{FD}_{\text {stab }}$ & NXFEM \\
\hline$\lambda_{\mathrm{C}}=0$ & $\lambda_{\mathrm{C}}=0$ & $\lambda_{\mathrm{C}}=1$ & $\gamma=10^{2}$ \\
\hline$\lambda_{\mathrm{M}}=1$ & $\begin{array}{c}\lambda_{\mathrm{M}}=1 \\
\varepsilon\end{array}$ & $\lambda_{\mathrm{M}}=\left\{\begin{array}{rll}1 & \text { in } & \Omega \backslash \omega_{h}^{n} \\
\varepsilon_{\mathrm{M}} & \text { in } & \omega_{h}^{n} \\
\varepsilon & & \end{array}\right.$ & $\begin{array}{c}\gamma_{\mathrm{v}}=\gamma_{\mathrm{p}}=10^{-2} \\
\gamma_{\mathrm{g}}=1\end{array}$ \\
\hline
\end{tabular}

Table 1: Free parameters of the numerical methods.

ods, the choice of the penalty parameter $\varepsilon$ and (for the latter) of the stabilization parameter $\varepsilon_{\mathrm{M}}$ requires a specific care which will be discussed in the next paragraphs.

\subsection{Example 1. Open valve}

As first example, we consider the heart-valve-inspired benchmark problem proposed in [34, 27, 31, 52. Due to the symmetric properties of the problem, only half of the domain is simulated with appropriate symmetry boundary conditions. The problem consists of one cantilevered elastic 


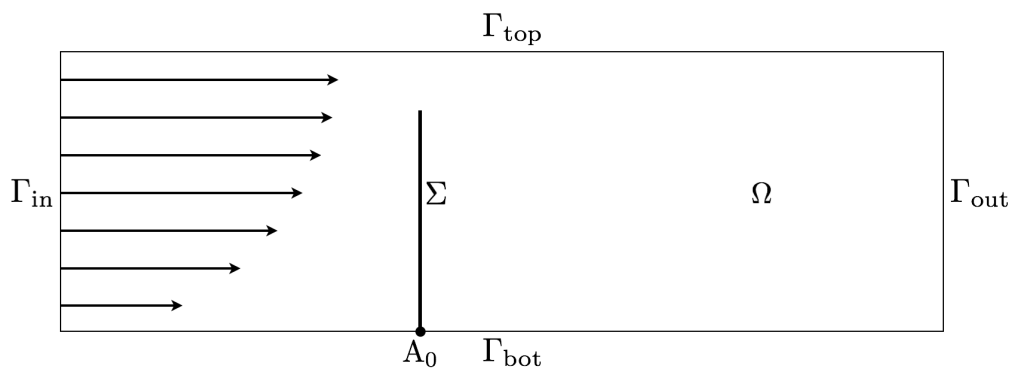

Figure 4: Geometric configuration and external boundary conditions.

beam immersed in a 2D-channel filled with an incompressible Newtonian fluid, as shown in Figure 4 .

The geometry of the fluid domain is given by $\Omega=[0,8] \times[0,0.805]$. The reference configuration of the solid is the segment with endpoints $(2,0)$ and $(2,0.7)$ (see Figure 4). The physical parameters for the fluid are $\rho^{\mathrm{f}}=100$ and $\mu=10$. For the solid, we have $\rho^{\mathrm{s}}=100, \epsilon=0.0212$, with Young's modulus $E=5.6 \times 10^{7}$ and Poisson's ratio $\nu=0.4$. All the units are given in the CGS units system.

As regards the boundary conditions for the fluid, a no-slip boundary condition is enforced on $\Gamma_{\text {bot }}$ while a symmetry boundary condition is imposed on $\Gamma_{\text {top }}$. Zero traction is enforced on the lateral boundary $\Gamma_{\text {out }}$. The velocity is prescribed on $\Gamma_{\text {in }}$, as a half parabolic profile whose maximum amplitude is defined by a positive time-dependent function $u_{\max }(t)$ given by

$$
u_{\max }(t)=5 \times(0.805)^{2} \times(\sin (2 \pi t)+1.1) .
$$

The beam is fully clamped at its bottom endpoint. The fluid and the solid are initially at rest.

\subsubsection{Spatial and temporal discretization}

A reference solution has been generated using Algorithm 1 with a high space-time grid resolution. The solid mesh is made of 59 segments and the body-fitted fluid mesh of 48706 quadrilaterals which are refined around the interface. The initial configuration of the fitted meshes is depicted in Figure 5a. Here, the fluid velocity and pressure fields are approximated using $\mathbb{Q}_{1}$ finite elements. The time step is $\tau=10^{-3}$ and the final time is $t=3$, corresponding to around 3 full cycles of oscillations of the structure. A snapshot of the resulting deformed mesh at time $t=0.5$ is shown in Figure 6a.

The first level of refinement for the unfitted meshes, which we refer to as $\mathrm{M}_{1}$, divides the fluid domain into 4096 triangular elements and the structure domain into 32 segments. The corresponding time step is $\tau=10^{-2}$. The two subsequent space-time grids, respectively noted $\mathrm{M}_{2}$ and $\mathrm{M}_{3}$, are uniform refinements of $\mathrm{M}_{1}$ with a factor of, respectively, 2 and 4 along the spatial and temporal directions. Hence, $\mathrm{M}_{2}$ is made of 16384 triangular elements and $\mathrm{M}_{3}$ of 65536 triangular elements. Their corresponding structure domains are respectively divided into 64 and 128 segments. The time steps are $\tau=5 \times 10^{-3}$ in $\mathrm{M}_{2}$ and $\tau=2.5 \times 10^{-3}$ in $\mathrm{M}_{3}$. For illustration purposes, $\mathrm{M}_{1}, \mathrm{M}_{2}$ and $\mathrm{M}_{3}$ at $t=0$ are shown in Figures $5 \mathrm{~b} 5 \mathrm{~d}$ and at $t=0.5$ in Figures 6b 6d (obtained with the NXFEM method).

\subsubsection{Comparison of results}

The values of the penalty parameter $\varepsilon$ for the FD method are equal to $10^{-5}$ with $\mathrm{M}_{1}, 10^{-6}$ with $\mathrm{M}_{2}$ and $10^{-6}$ with $\mathrm{M}_{3}$. The motivation of these choices will be discussed in Section 4.1 .3 below. 


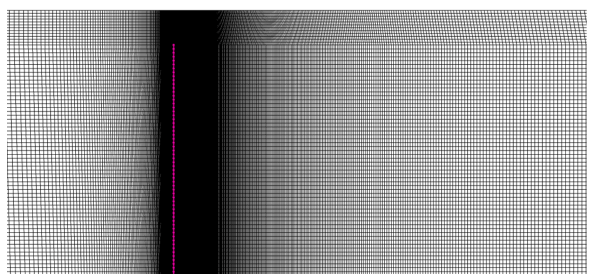

(a) Fitted meshes - Reference.

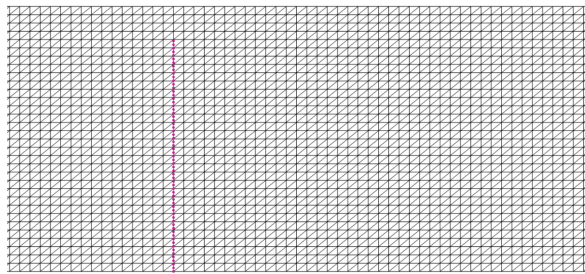

(c) Unfitted meshes - $\mathrm{M}_{2}$.

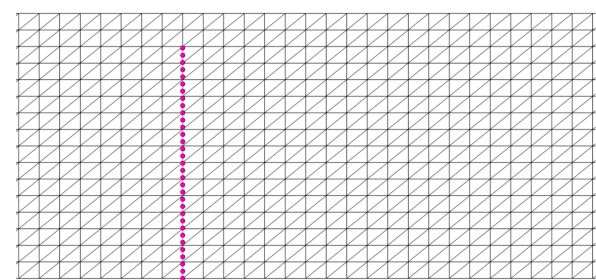

(b) Unfitted meshes - $\mathrm{M}_{1}$.

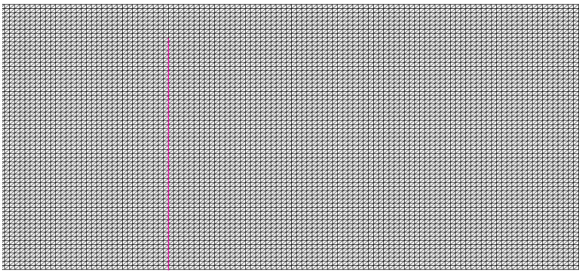

(d) Unfitted meshes - $\mathrm{M}_{3}$.

Figure 5: Zoom on the fluid and solid meshes at time $t=0$.

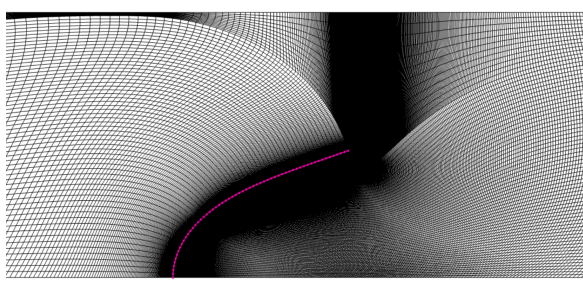

(a) Fitted meshes - Reference.

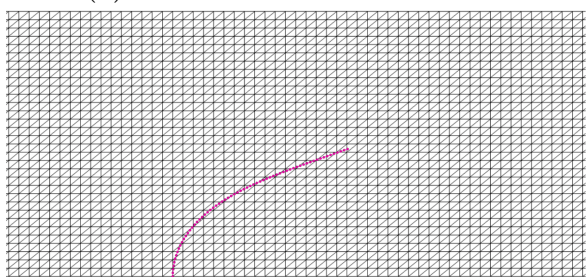

(c) Unfitted meshes - $\mathrm{M}_{2}$.

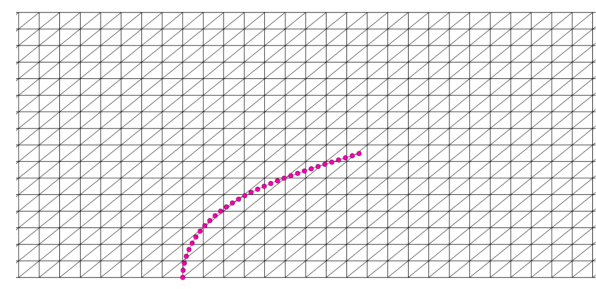

(b) Unfitted meshes $-\mathrm{M}_{1}$.

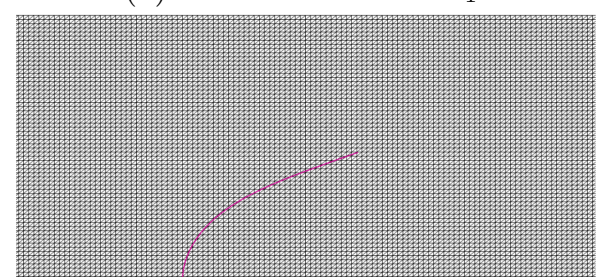

(d) Unfitted meshes - $\mathrm{M}_{3}$.

Figure 6: Zoom on the fluid and solid meshes at time $t=0.5$.

For this example, the results obtained with the $\mathrm{FD}_{\text {stab }}$ variant method are very close to those obtained with the FD method and, hence, are omitted.

Figures 7 and 8 present snapshots of the interface location and fluid velocity magnitude near the leaflet at $t=0.5$ for the three levels of refinement and for the NXFEM and FD methods, respectively. Overall, a good agreement of the velocity field with the ALE method is already observed for the coarsest grid $\mathrm{M}_{1}$. Note that the correct computation of the induced vortex after the leaflet requires more refinement and it is better captured by the NXFEM method.

The results for the pressure are given in Figures 9 and 10, respectively for the NXFEM and FD methods. The NXFEM method gets the correct shape of the pressure field, even with the coarsest grid resolution. After refinement, the agreement with the ALE method is very good in both approaches. For the FD method, comparisons between $\mathrm{M}_{1}$ and $\mathrm{M}_{3}$ clearly indicates that 


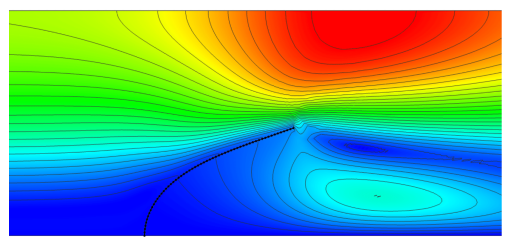

(a) ALE method - Reference.

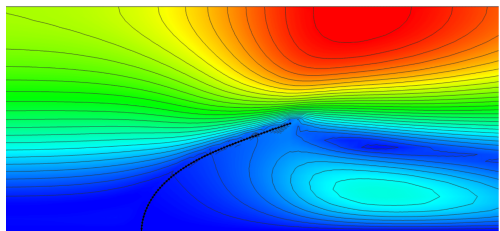

(c) NXFEM method - $\mathrm{M}_{2}$.

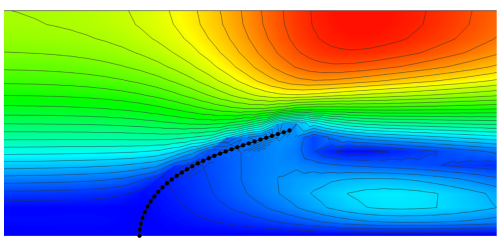

(b) NXFEM method - $M_{1}$.

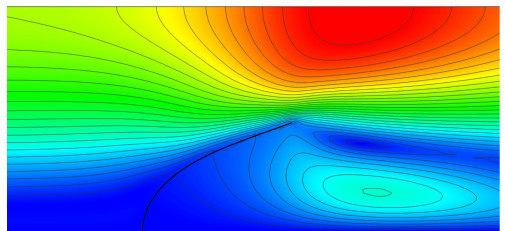

(d) NXFEM method - $M_{3}$.

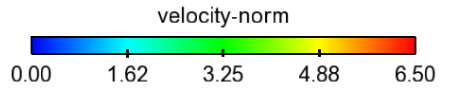

Figure 7: Snapshots of the fluid velocity magnitude at $t=0.5$ obtained with NXFEM.

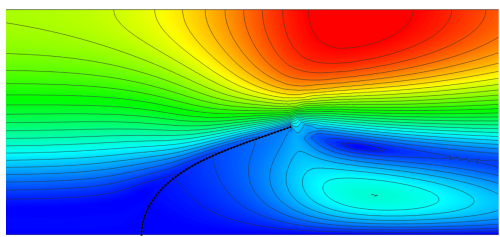

(a) ALE method - Reference.

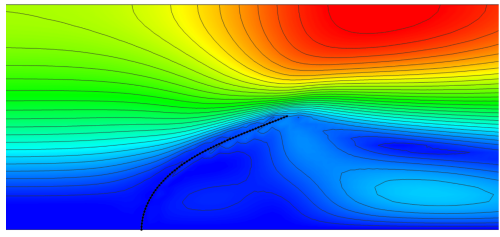

(c) FD method $-\mathrm{M}_{2}$.

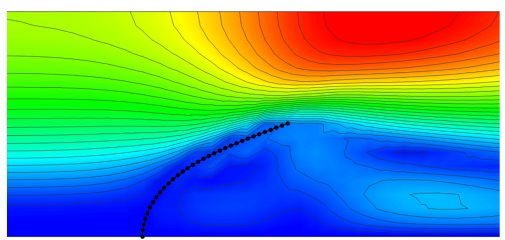

(b) FD method $-M_{1}$.

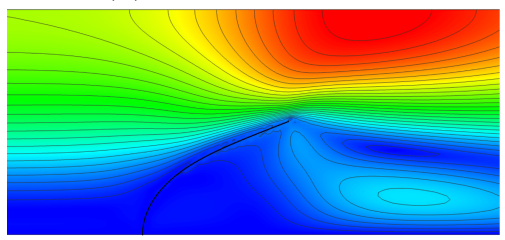

(d) FD method $-\mathrm{M}_{3}$.

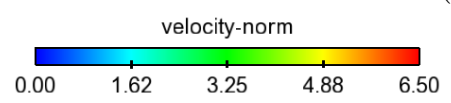

Figure 8: Snapshots of the fluid velocity magnitude at $t=0.5$ obtained with FD.

mesh refinement improves the results.

Figure 11 reports a comparison of the time history of the $x$-solid displacement at the upper leaflet tip obtained with the NXFEM and FD methods. The NXFEM method shows better agreement with the ALE method. Nevertheless, the overall agreement of the FD methods is satisfying for the $\mathrm{M}_{2}$ and $\mathrm{M}_{3}$ refinement levels.

Similar observations can be made from Figure 12 , which reports the time history of the $y$-solid displacement of the upper leaflet tip. Note that the convergence of the FD method towards the reference solution is clearly visible, but slower than for the NXFEM method. 


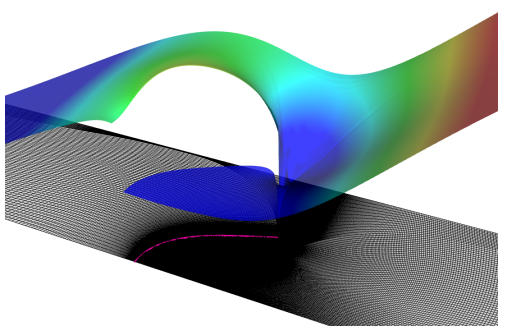

(a) ALE method - Reference.

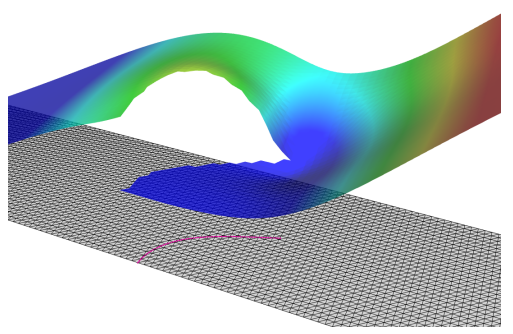

(c) NXFEM method $-\mathrm{M}_{2}$.

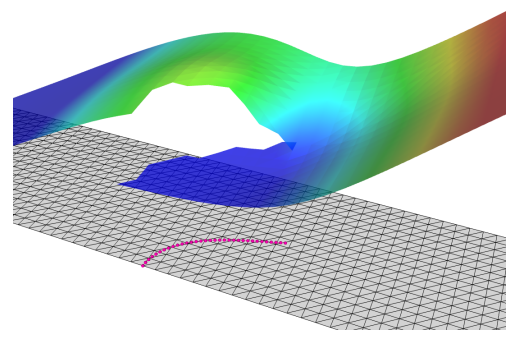

(b) NXFEM method - $\mathrm{M}_{1}$.

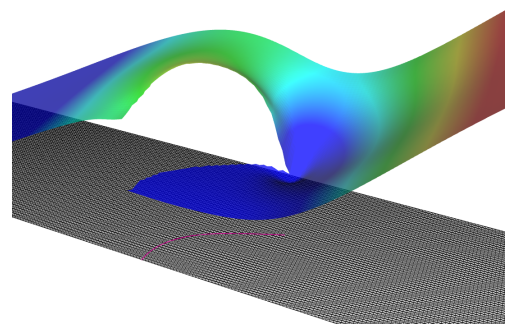

(d) NXFEM method - $M_{3}$.

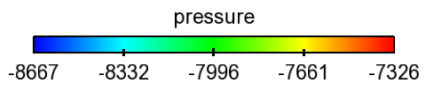

Figure 9: Snapshots of the fluid elevated pressure at $t=0.5$ obtained with NXFEM.

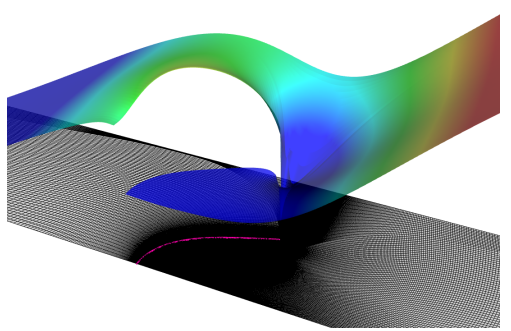

(a) ALE method - Reference.

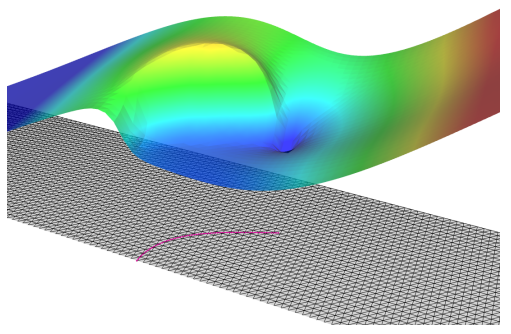

(c) FD method $-\mathrm{M}_{2}$.

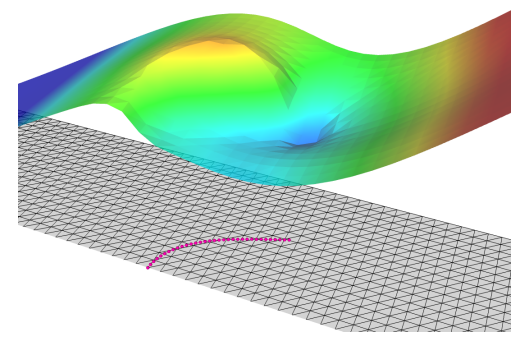

(b) FD method $-\mathrm{M}_{1}$.

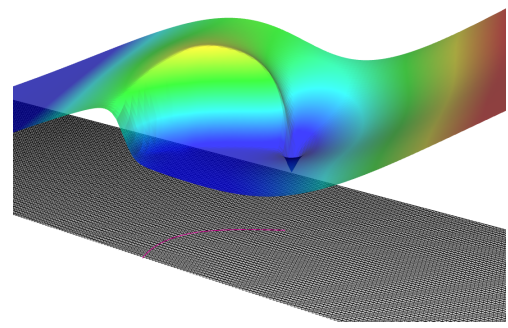

(d) FD method $-\mathrm{M}_{3}$.

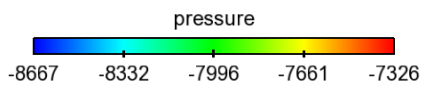

Figure 10: Snapshots of the fluid elevated pressure at $t=0.5$ obtained with FD. 


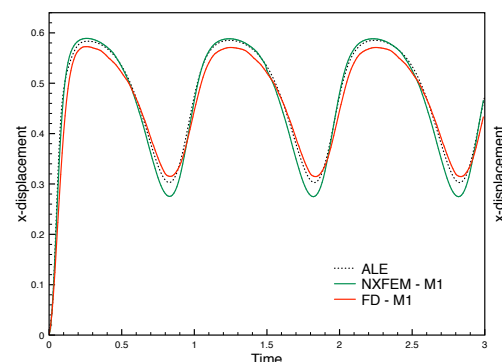

(a) $M_{1}$ vs. Reference.

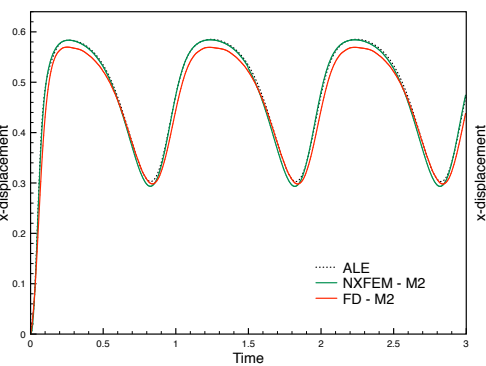

(b) $\mathrm{M}_{2}$ vs. Reference.

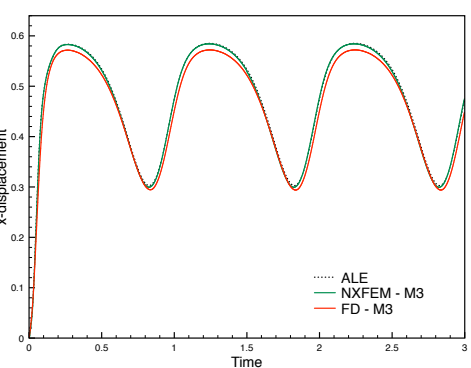

(c) $\mathrm{M}_{3}$ vs. Reference.

Figure 11: Time history of the $x$-displacement of the upper leaflet tip.

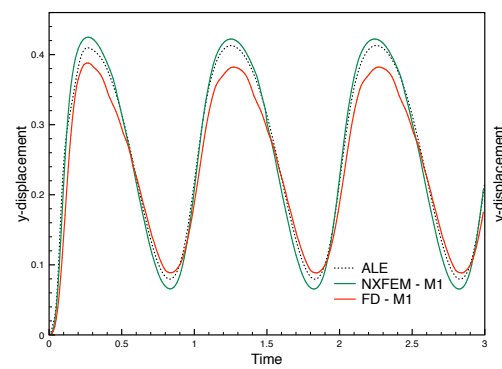

(a) $M_{1}$ vs. Reference.

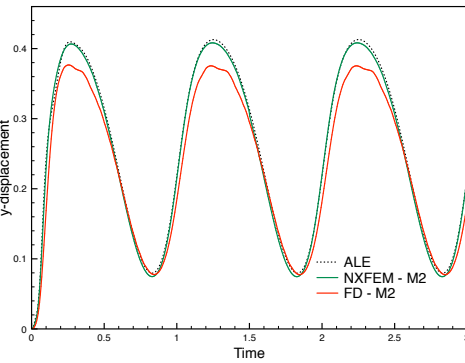

(b) $\mathrm{M}_{2}$ vs. Reference.

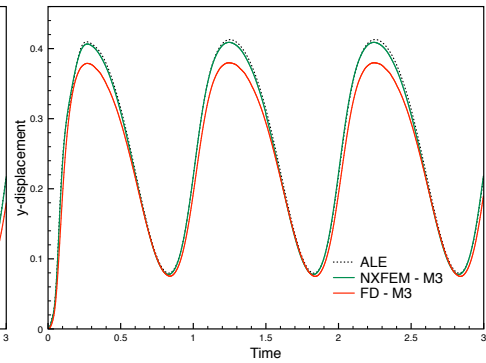

(c) $\mathrm{M}_{3}$ vs. Reference.

Figure 12: Time history of the $y$-displacement of the upper leaflet tip.

\subsubsection{Choice of the penalty parameters for the fictitious domain methods}

For the FD method introduced in Algorithm 2, the critical parameter to fix is the penalization coefficient $\varepsilon$. The choice of the values of penalty parameters is usually delicate and arbitrary (see, e.g., [53, 42 ). Here, we present the workflow we carried on to determine a range of admissible values for the penalization coefficient $\varepsilon$. Our approach is similar to the one used in [53]: a given simulation has been run with a large range of values for the penalty parameter $\varepsilon$. The value is initially $10^{-1}$ and is reduced until the convergence is lost due to ill-conditioning problems of the system. A physical value, characterizing the current case, is then computed with respect to the time. This convergence study is done on for each level of refinement.

The physical value of interest here is the displacement magnitude of the immersed upper leaflet tip. The set of values used for the $\varepsilon$ are

$$
\varepsilon= \begin{cases}\left\{10^{-i}, i=1 \ldots 6\right\}, & \text { for } \mathrm{M}_{1}, \\ \left\{10^{-i}, i=1 \ldots 7\right\}, & \text { for } \mathrm{M}_{2}, \\ \left\{10^{-i}, i=1 \ldots 7\right\}, & \text { for } \mathrm{M}_{3},\end{cases}
$$

and the corresponding results for the levels of refinement $M_{1}, M_{2}$ and $M_{3}$ are respectively shown in Figures $13 \mathrm{a} 13 \mathrm{c}$

As expected, all the curves have the same behavior, independently of the penalization or the refinement of the meshes. The penalty parameter $\varepsilon$ has an impact on the amplitude of the displacement: when the penalization is stronger, the different curves convergence towards the same value. As the penalization of the immersed structure appears in the fluid problem under 


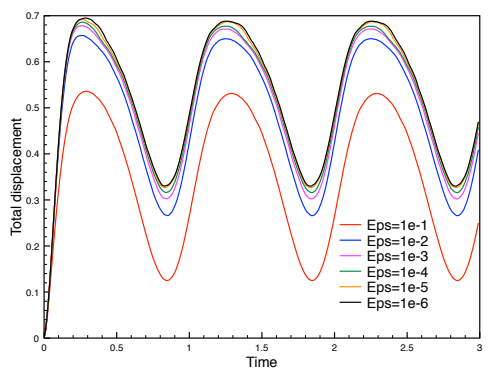

(a) $\mathrm{M}_{1}$

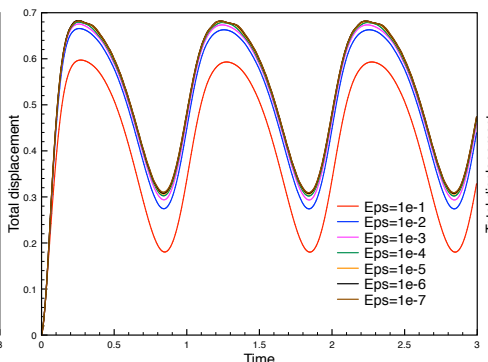

(b) $\mathrm{M}_{2}$.

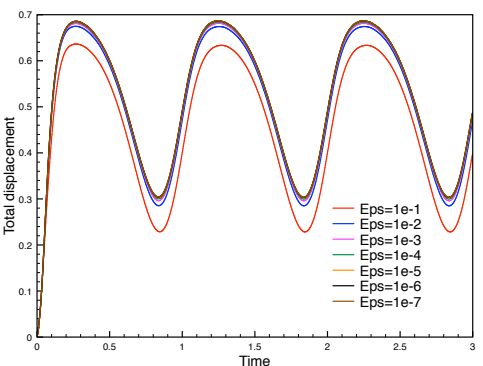

(c) $\mathrm{M}_{3}$.

Figure 13: Convergence study of the penalty parameter $\varepsilon$.

the form $\frac{1}{\varepsilon}$, a small penalty parameter may induce ill-conditioning of the system and then, low convergence rate. This is why we assumed that, once a good over-all convergence has been achieved and the results are independent of the values of $\varepsilon$, it is no more useful to further reduce it.

\subsection{Example 2. Closed valve}

As second example, we consider a benchmark problem mimicking the behavior of a closed valve (see, e.g., 34, 49]). The main difficulty here are the correct evaluation of the pressure jump and the conservation of mass across the valve.

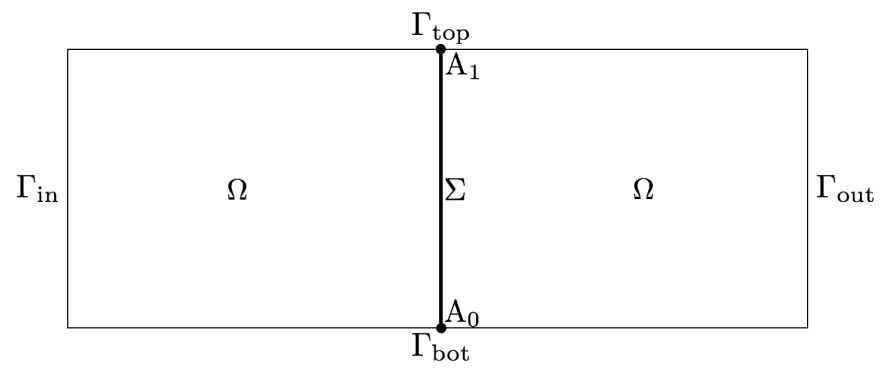

Figure 14: Geometric configuration and external boundary conditions.

The problem consists of an elastic beam clamped on its endpoints and immersed in a 2Dchannel filled with an incompressible Newtonian fluid, as shown in Figure 4. The geometry of the fluid domain is given by $\Omega=[0,4] \times[0,1]$. The reference configuration for the solid is defined as the segment with endpoints $(2,0)$ and $(2,1)$ (see Figure 14). The physical parameters for the fluid are $\rho^{\mathrm{f}}=100$ and $\mu=10$. For the solid, we have $\rho^{\mathrm{s}}=100, \epsilon=0.0212$, with Young's modulus $E=5.6 \times 10^{7}$ and Poisson's ratio $\nu=0.4$. All the units are given in the CGS units system.

As regards the boundary conditions for the fluid, a no-slip boundary condition is enforced on $\Gamma_{\text {bot }}$ and $\Gamma_{\text {top }}$. Zero traction is enforced on the lateral boundary $\Gamma_{\text {out }}$, while on $\Gamma_{\text {in }}$ traction is imposed in terms of the following time-dependent pressure

$$
p_{\text {in }}(t)=3 \times 10^{5} \times \operatorname{atanh}(10 \times t) .
$$

The structure is fully clamped at its endpoints $A_{0}$ and $A_{1}$. The fluid and the solid are initially at rest. As the prescribed pressure builds up, the solid starts to bend and elongate. After this 
brief transition phase, the system reaches a steady state with a pressure jump across the interface. The fluid and solid velocities vanish and the pressure is a piecewise constant function taking the value $3 \times 10^{5}$ and zero on each side of the interface, respectively.

\subsubsection{Spatial and temporal discretization}

A reference solution has been generated using Algorithm 1 with a high space-time grid resolution. The solid mesh is made of 80 segments and the body-fitted fluid mesh of 25600 quadrilaterals. The initial configuration of the fitted meshes is depicted in Figure $15 \mathrm{a}$

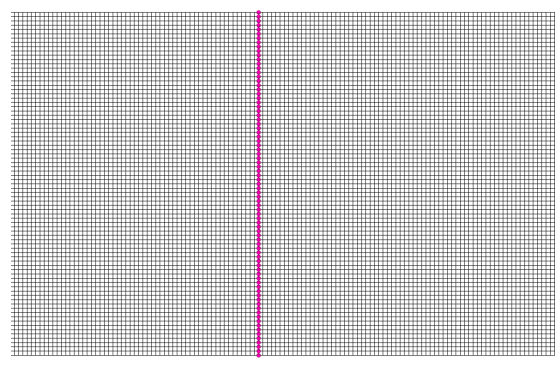

(a) Fitted meshes - Reference.

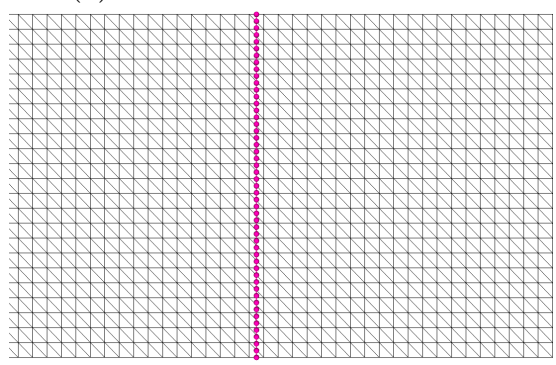

(c) Unfitted meshes - $\mathrm{M}_{2}$.

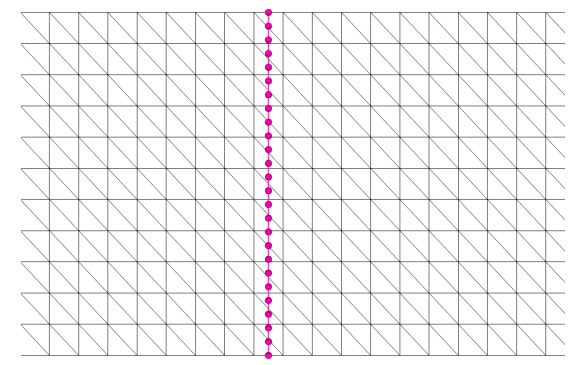

(b) Unfitted meshes - $\mathrm{M}_{1}$.

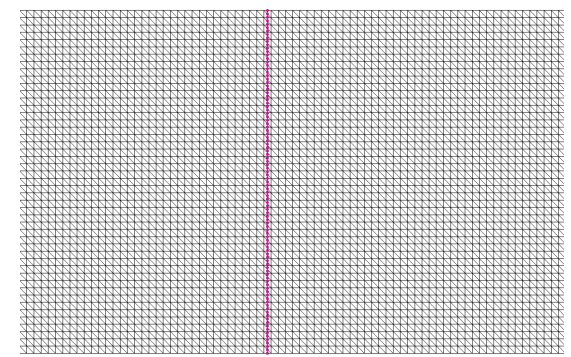

(d) Unfitted meshes $-\mathrm{M}_{3}$.

Figure 15: Zoom on the fluid and solid meshes at time $t=0$.

Here, the fluid velocity and pressure fields are approximated using $\mathbb{Q}_{1}$ finite elements. The time step is $\tau=10^{-3}$ and the final time is $t=3$, corresponding to the time required by the structure to reach its steady state. A snapshot of the resulting deformed mesh at time $t=0.125$ is shown in Figure $16 \mathrm{a}$.

The first level of refinement for the unfitted meshes, $\mathrm{M}_{1}$, divides the fluid domain into 1034 triangular elements and the structure domain into 25 segments. The corresponding time step is $\tau=2 \times 10^{-3}$. As in the previous example, the two subsequent space-time grids are uniform refinements of $\mathrm{M}_{1}: \mathrm{M}_{2}$ is made of 4370 triangular elements and $\mathrm{M}_{3}$ of 17954 triangular elements. Their corresponding structure domains are respectively divided into 50 and 100 segments. The time steps are $\tau=10^{-3}$ in $\mathrm{M}_{2}$ and $\tau=5 \times 10^{-4}$ in $\mathrm{M}_{3}$. For illustration purposes, $\mathrm{M}_{1}, \mathrm{M}_{2}$ and $\mathrm{M}_{3}$ at $t=0$ are shown in Figures $15 \mathrm{~b} 15 \mathrm{~d}$ and at $t=0.125$ in Figures $16 \mathrm{~b} 16 \mathrm{~d}$ (obtained with the NXFEM method).

\subsubsection{Comparison of results}

For all the refinement levels, we have taken $\varepsilon=10^{-5}$ in the $\mathrm{FD}$ and $\mathrm{FD}_{\text {stab }}$ methods. In the latter, the stabilization parameter is set to $\varepsilon_{M}=10^{-4}$. The motivation of these choices will be 


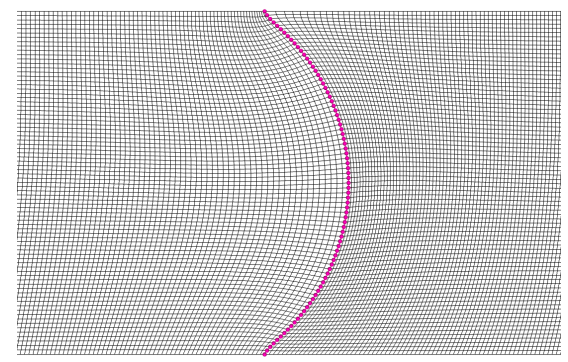

(a) Fitted meshes - Reference.

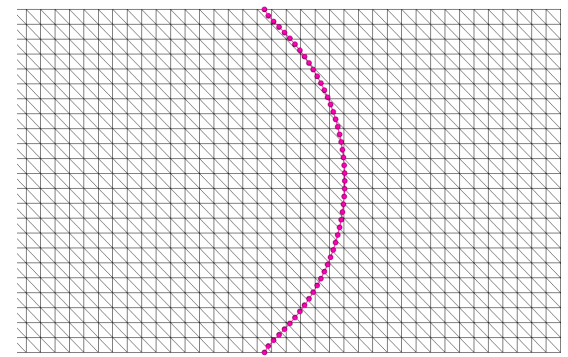

(c) Unfitted meshes - $\mathrm{M}_{2}$.

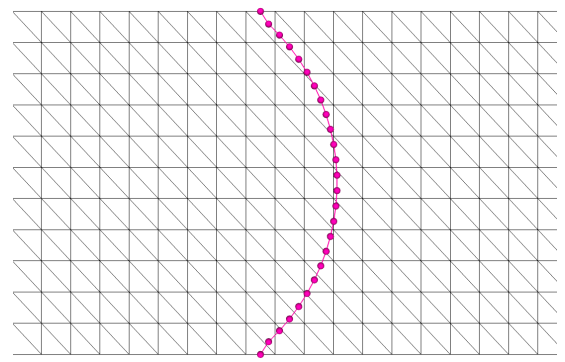

(b) Unfitted meshes - $\mathrm{M}_{1}$.

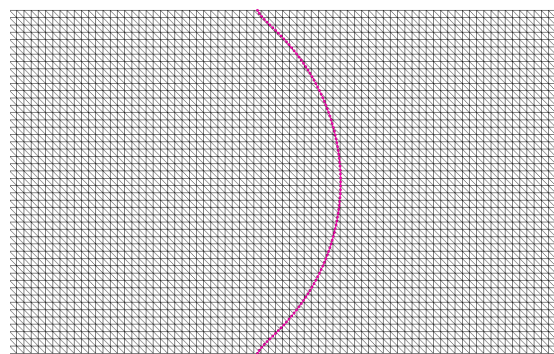

(d) Unfitted meshes - $\mathrm{M}_{3}$.

Figure 16: Zoom on the fluid and solid meshes at time $t=0.125$.

discussed in Section 4.2 .3 below.

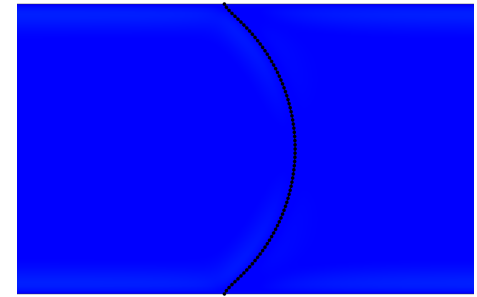

(a) ALE method - Reference.

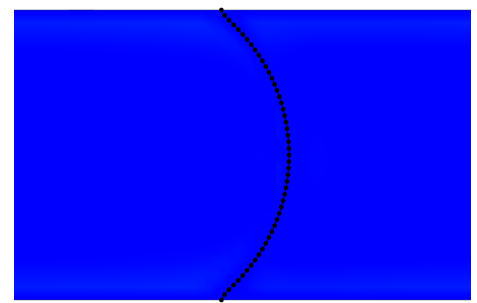

(c) NXFEM method $-\mathrm{M}_{2}$.

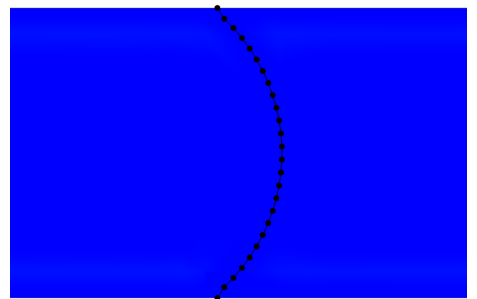

(b) NXFEM method - $\mathrm{M}_{1}$.

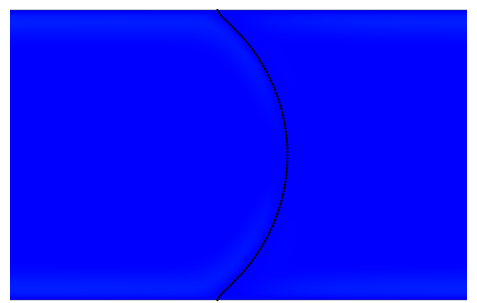

(d) NXFEM method $-\mathrm{M}_{3}$.

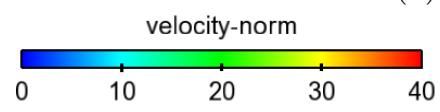

Figure 17: Snapshots of the fluid velocity magnitude at $t=0.125$ obtained with NXFEM.

Figures 17,19 present, respectively for the NXFEM, FD and FD $_{\text {stab }}$ methods, the snapshots 


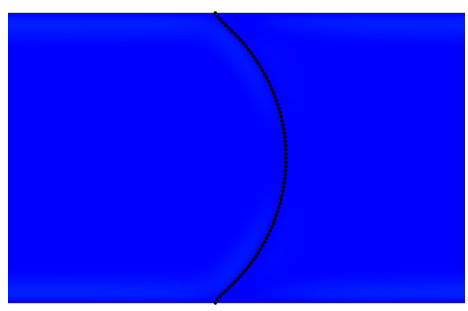

(a) ALE method - Reference.

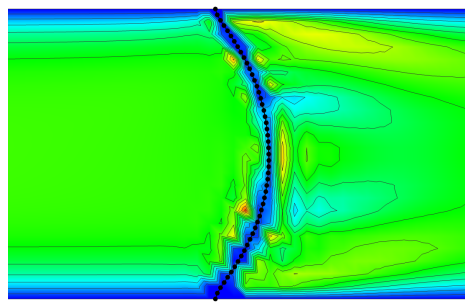

(c) FD method $-\mathrm{M}_{2}$.

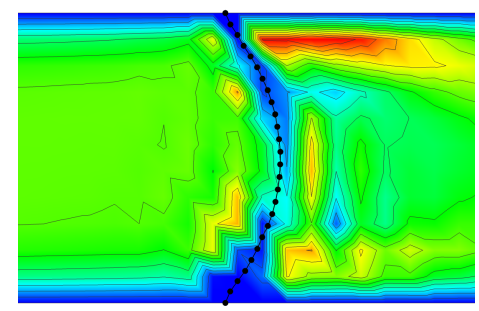

(b) FD method - M .

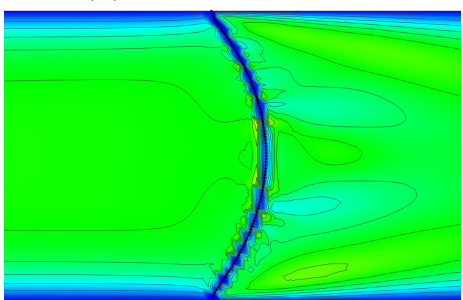

(d) FD method $-\mathrm{M}_{3}$

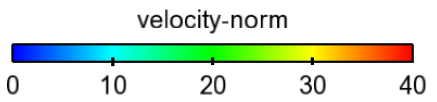

Figure 18: Snapshots of the fluid velocity magnitude at $t=0.125$ obtained with FD.

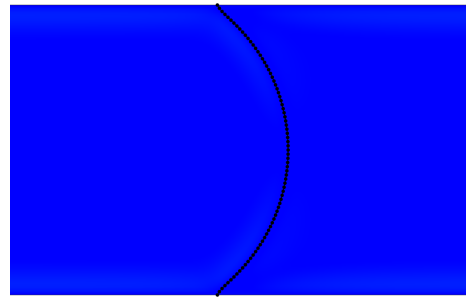

(a) ALE method - Reference.

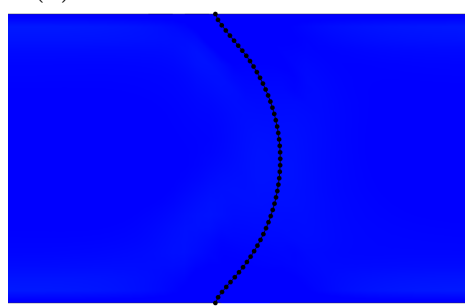

(c) $\mathrm{FD}_{\text {stab }}$ method $-\mathrm{M}_{2}$.

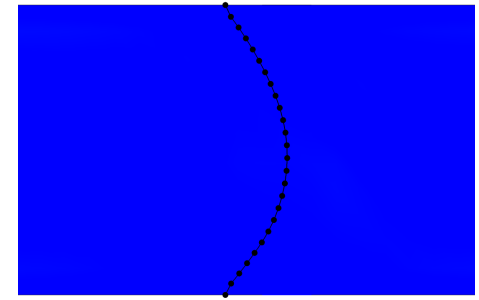

(b) $\mathrm{FD}_{\text {stab }}$ method $-\mathrm{M}_{1}$.

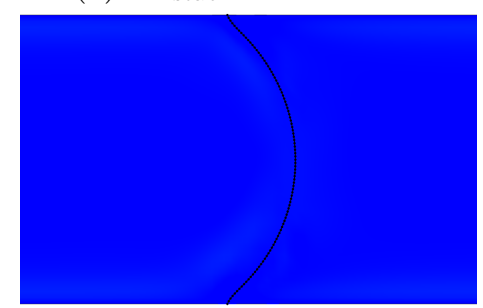

(d) $\mathrm{FD}_{\text {stab }}$ method $-\mathrm{M}_{3}$.

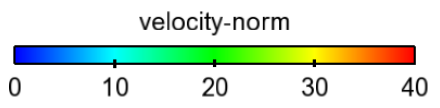

Figure 19: Snapshots of the fluid velocity magnitude at $t=0.125$ obtained with $\mathrm{FD}_{\mathrm{stab}}$.

of the interface location and the fluid velocity magnitude near the leaflet at $t=0.125$ for the three levels of refinement. The NXFEM method retrieves the negligible velocity field of the reference ALE solution in all the grid refinement levels. The color palette has been kept the same for the 


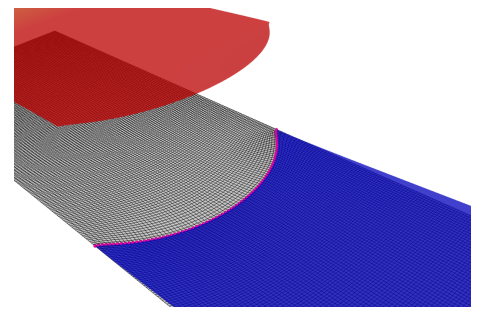

(a) ALE method - Reference.

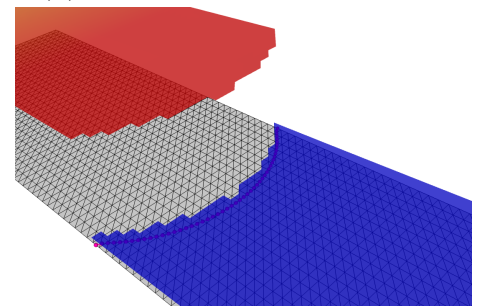

(c) NXFEM method - $\mathrm{M}_{2}$.

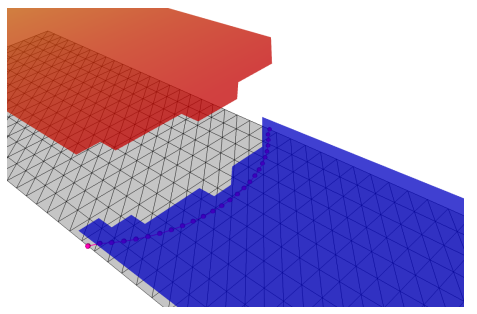

(b) NXFEM method - $M_{1}$.

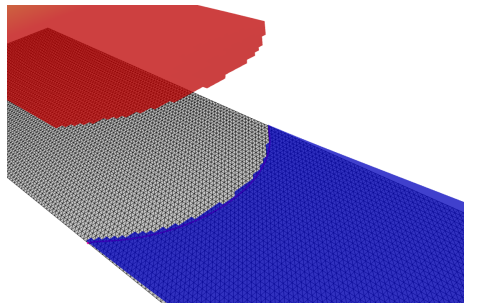

(d) NXFEM method - $\mathrm{M}_{3}$.

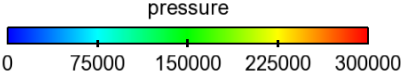

Figure 20: Snapshots of the fluid elevated pressure at $t=0.125$ obtained with NXFEM.

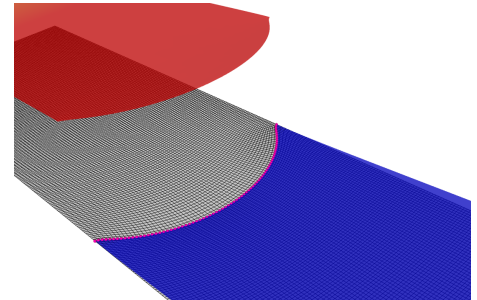

(a) ALE method - Reference.

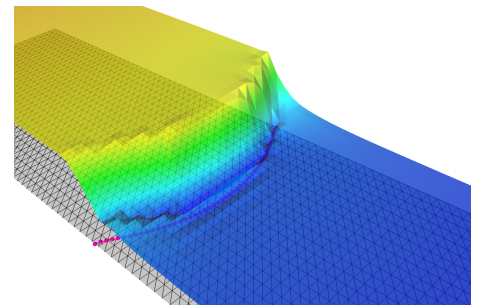

(c) FD method $-\mathrm{M}_{2}$.

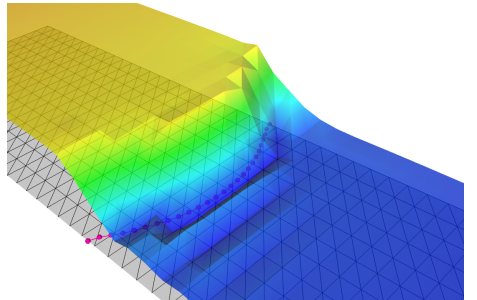

(b) FD method - $\mathrm{M}_{1}$.

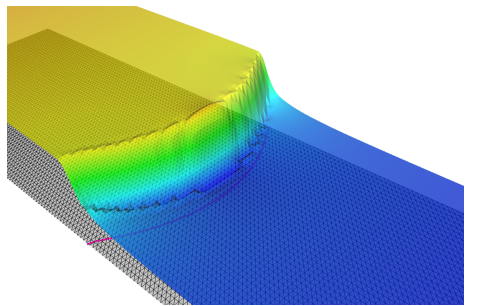

(d) FD method - $\mathrm{M}_{3}$.

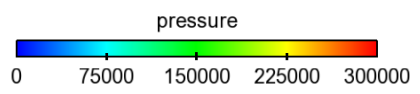

Figure 21: Snapshots of the fluid elevated pressure at $t=0.125$ obtained with FD.

three figures in order to highlight the loss of mass featured by the FD method. In Figure 18, no matter what level of refinement is used, a significant spurious flow is observed through the channel. This phenomenon is an important limitation of the FD method with continuous pressure approximations (see, e.g., 4, 34]), which guarantees global but not local mass conservation (i.e., 


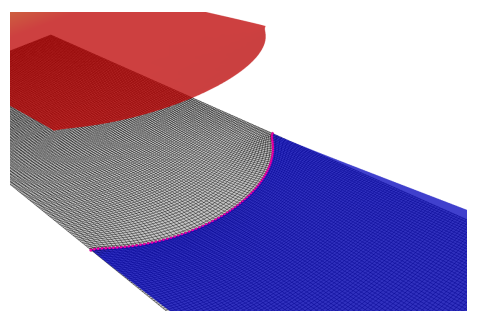

(a) ALE method - Reference.

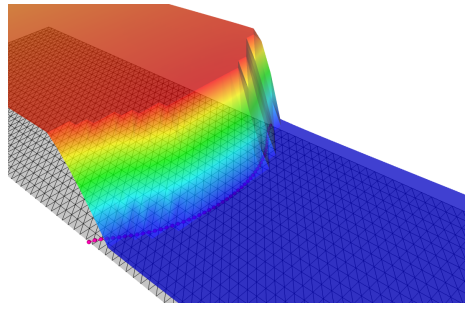

(c) $\mathrm{FD}_{\text {stab }}$ method $-\mathrm{M}_{2}$.

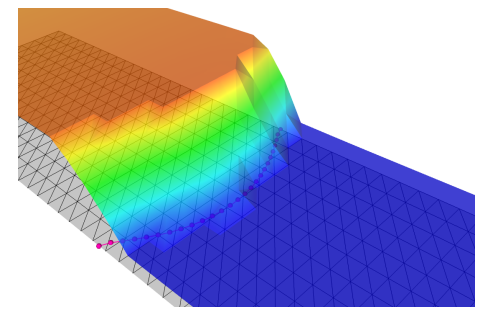

(b) $\mathrm{FD}_{\text {stab }}$ method $-\mathrm{M}_{1}$.

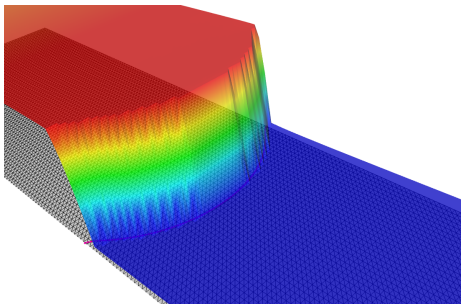

(d) $\mathrm{FD}_{\text {stab }}$ method $-\mathrm{M}_{3}$.

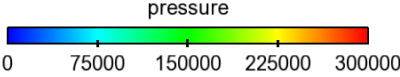

Figure 22: Snapshots of the fluid elevated pressure at $t=0.125$ obtained with $\mathrm{FD}_{\text {stab }}$.

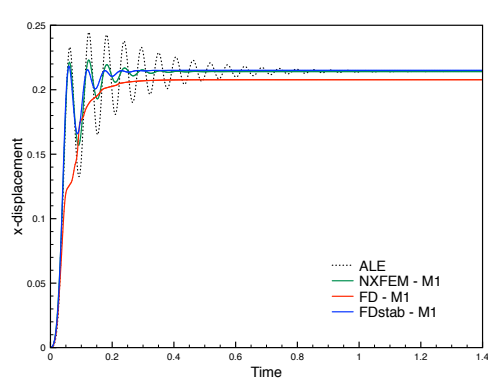

(a) $M_{1}$ vs. Reference.

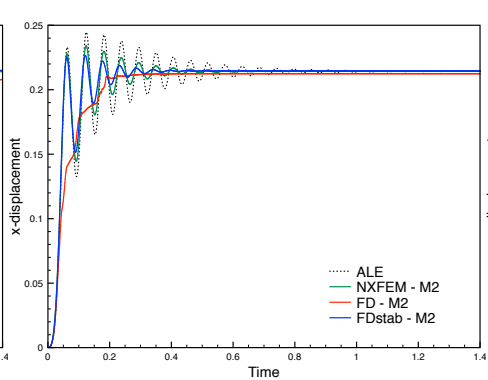

(b) $\mathrm{M}_{2}$ vs. Reference.

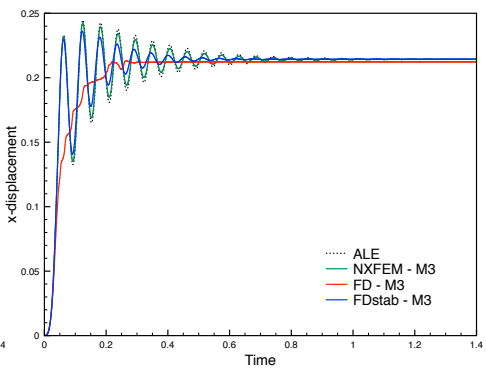

(c) $M_{3}$ vs. Reference.

Figure 23: Time history of the $x$-displacement of the valve mid-point.

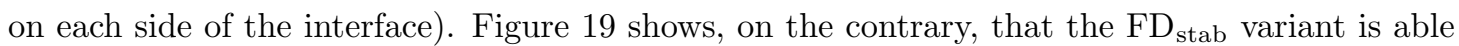
to significantly reduce this leak across the interface, which highlights the benefits of the modified SUPG/PSPG and grad-div stabilizations proposed in [34].

The results for the pressure are reported in Figures 20 22, respectively for the NXFEM, FD and $\mathrm{FD}_{\text {stab }}$ methods. Once more, the NXFEM method gets the correct pressure jump, particularly with the grid refinement levels $\mathrm{M}_{2}$ and $\mathrm{M}_{3}$. On the opposite, the FD method struggles to correctly compute the pressure jump, which is largely under-estimated compared to the reference solution. The $\mathrm{FD}_{\text {stab }}$ method shows a very good correction of the pressure jump across the valve compared to the FD method. Moreover, the fact that the prediction of the pressure jump improves with the refinement is much more striking in Figure 22

Figure 23 reports a comparison of the time history of the $x$-solid displacement of the valve

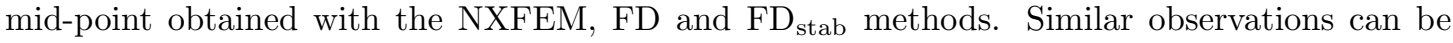
made from these results. The NXFEM and $\mathrm{FD}_{\text {stab }}$ methods shows better agreement with the 
reference solution than with the FD method. One particular point of interest is the computation of the dynamics of the valve, characterized by the oscillations of the $x$-value around the reference steady state. The FD method is clearly unable to compute the initial dynamics of the valve, no matter what level of refinement is used. On the contrary, the NXFEM and FD stab predict the dynamics of the closed and show convergence to the reference after grid refinement.

\subsubsection{Choice of the penalty parameters for the fictitious domain methods}

As explained in Subsection 4.1.3, we carried on the same convergence study with respect to the penalty parameter $\varepsilon$, appearing in the FD method. The physical value of interest here is the $x$-displacement of the valve mid-point. For each level of refinement, the case has been run several times with a large panel of values for $\varepsilon$. The same behaviors as the ones described in Section 4.1.3 have also been observed here. The chosen values of $\varepsilon$ are based on the previous mentioned criteria of convergence.

For the sake of illustration, the interfacial zone in which the modified $\mathrm{FD}_{\text {stab }}$ acts, is depicted at $t=0.125$ in Figures 24a $24 \mathrm{c}$, respectively for the meshes $\mathrm{M}_{1}, \mathrm{M}_{2}$ and $\mathrm{M}_{3}$. In order to choose

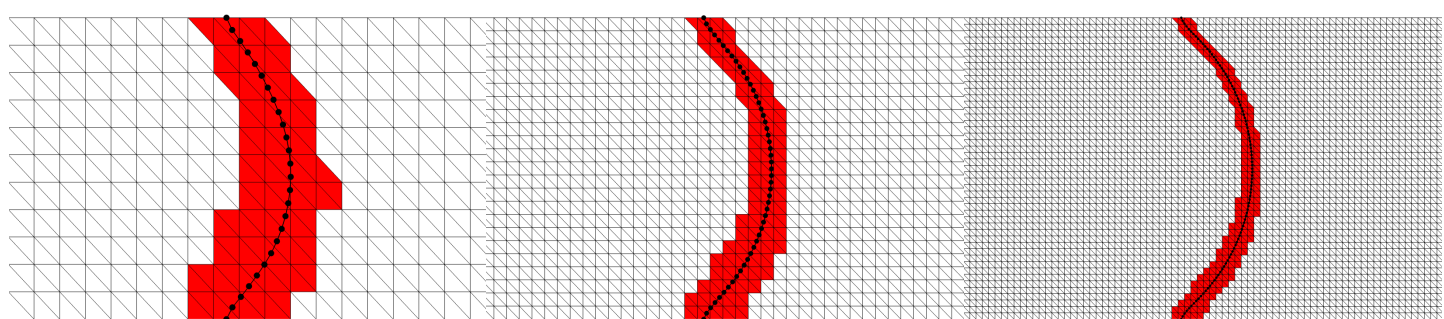

(a) $\mathrm{M}_{1}$.

(b) $\mathrm{M}_{2}$.

(c) $\mathrm{M}_{3}$.

Figure 24: The neighborhood $\omega_{h}^{n}$ at time $t=0.125$.

the values of $\varepsilon_{\mathrm{M}}$, the same kind of convergence study as the one described in Subsection 4.1 .3 for $\varepsilon$ has been carried on. For each case $M_{1}, M_{2}$ and $M_{3}, \varepsilon$ is equal to the value given by the convergence study and the case is run several times with a large range of values for the user-defined parameter $\varepsilon_{\mathrm{M}}$. The set of values used for $\varepsilon_{\mathrm{M}}$ are:

$$
\varepsilon_{\mathrm{M}}= \begin{cases}\left\{10^{-i}, i=0 \ldots 4\right\}, & \text { for } \mathrm{M}_{1}, \\ \left\{10^{-i}, i=0 \ldots 4\right\}, & \text { for } \mathrm{M}_{2} \\ \left\{10^{-i}, i=0 \ldots 4\right\}, & \text { for } \mathrm{M}_{3}\end{cases}
$$

The lowest value, which do not compromise the convergence of the simulation, is chosen.

\subsection{Example 3. Vesicle in lid-driven cavity flow}

As last example, we consider the lid-driven cavity benchmark problem (inspired from, e.g., 44, 50, 29, 22]) with an immersed elastic circle, as shown in Figure 25.

The geometry of the fluid domain is given by $\Omega=[0,0] \times[1,1]$. The reference solid configuration is a circle of radius $R=0.2$ centred at $(0.6,0.5)$ (see Figure 25). The physical parameters for the fluid are $\rho^{\mathrm{f}}=100$ and $\mu=10$. For the solid, we have $\rho^{\mathrm{s}}=100, \epsilon=0.0212$, with Young's modulus $E=5.6 \times 10^{3}$ and Poisson's ratio $\nu=0.4$. All the units are given in the CGS units system.

$\mathrm{RR} \mathrm{n}^{\circ} 9151$ 


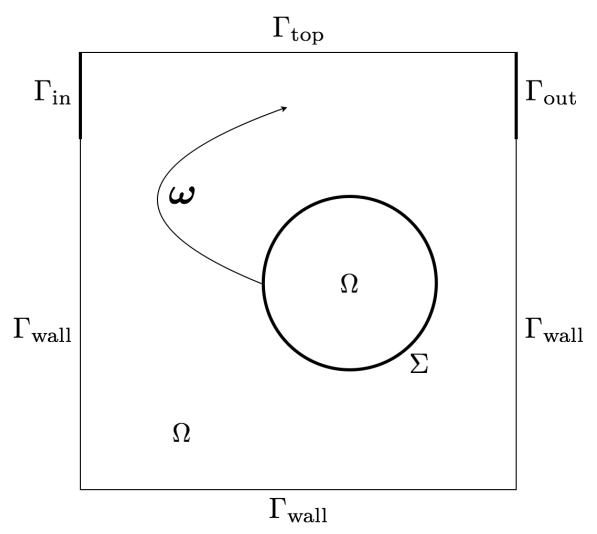

Figure 25: Geometric configuration and external boundary conditions.

As regards the boundary conditions for the fluid, a no-slip boundary condition is enforced on $\Gamma_{\text {bot }}$ and $\Gamma_{\text {wall }}$. Zero traction is enforced on the lateral boundaries $\Gamma_{\text {in }}$ and $\Gamma_{\text {out }}$. The velocity is prescribed on $\Gamma_{\text {top }}$, as a positive velocity whose amplitude is constant and equal to 1 . Both the fluid and the solid are initially at rest.

\subsubsection{Spatial and temporal discretization}

The reference ALE solution has been generated using a solid mesh made of 200 segments and a body-fitted fluid mesh of 46078 triangles. The initial configuration of the fitted meshes is depicted in Figure 26a. The time step is $\tau=2.5 \times 10^{-3}$ and the final time is $t=10$, approximately corresponding to a full turn of the vesicle. A snapshot of the resulting deformed mesh at time $t=6$ is shown in Figure 27a. We consider that $t=6$ is the upper limit where we can still refer to the ALE solution as the reference (close to the break-down of the mesh motion procedure).

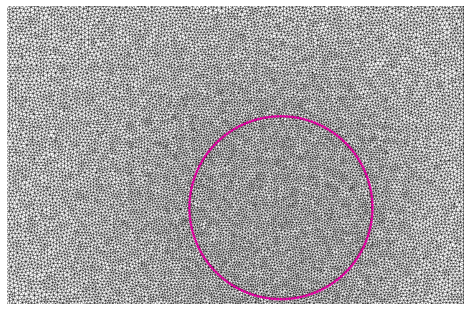

(a) Fitted meshes - Reference.

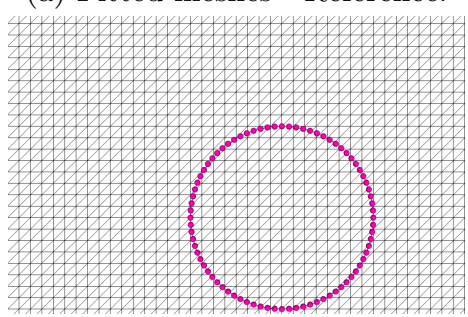

(c) Unfitted meshes $-\mathrm{M}_{2}$.

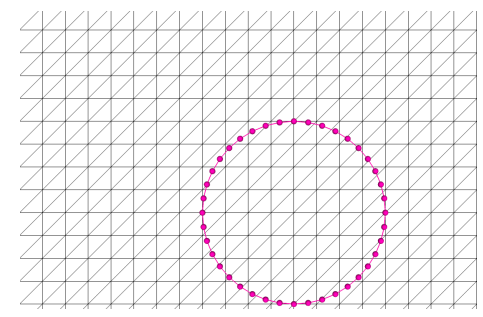

(b) Unfitted meshes - $\mathrm{M}_{1}$.

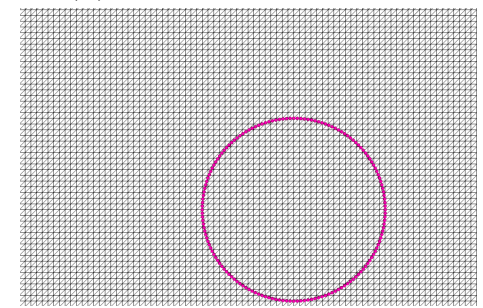

(d) Unfitted meshes - $\mathrm{M}_{3}$.

Figure 26: Zoom on the fluid and solid meshes at time $t=0$. 


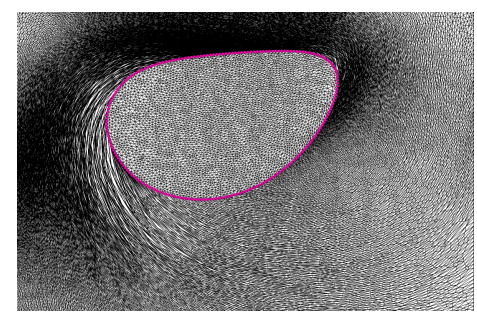

(a) Fitted meshes - Reference.

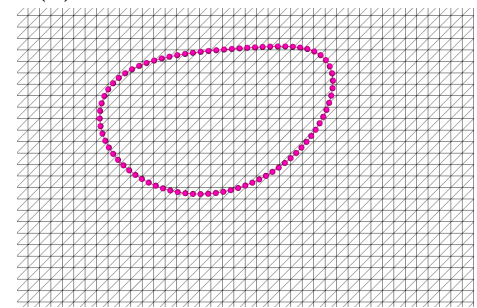

(c) Unfitted meshes - $\mathrm{M}_{2}$.

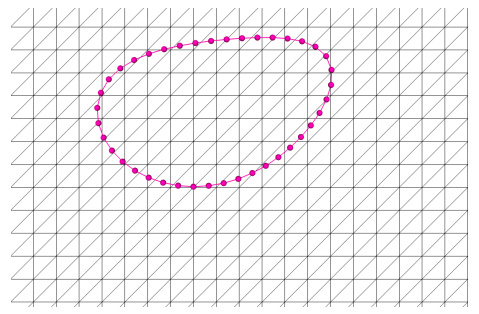

(b) Unfitted meshes - $\mathrm{M}_{1}$.

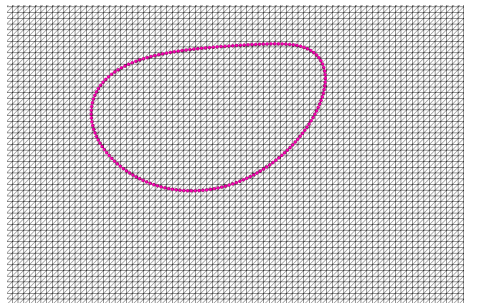

(d) Unfitted meshes - $\mathrm{M}_{3}$.

Figure 27: Zoom on the fluid and solid meshes at time $t=6$.

The first level of refinement for the unfitted meshes, $M_{1}$, divides the fluid domain into 800 triangular elements and the structure domain into 40 segments. The corresponding time step is $\tau=10^{-2}$. As in two previous test cases, the two subsequent space-time grids, denoted by $\mathrm{M}_{2}$ and $M_{3}$ respectively, are uniform refinements of $M_{1}$. Thus, $M_{2}$ is made of 3200 triangular elements and $\mathrm{M}_{3}$ of 12800 triangular elements. Their corresponding structure domains are divided into 80 and 160 segments respectively. The time steps are $\tau=5 \times 10^{-3}$ in $\mathrm{M}_{2}$ and $\tau=2.5 \times 10^{-3}$ in $\mathrm{M}_{3}$. For illustration purposes, $\mathrm{M}_{1}, \mathrm{M}_{2}$ and $\mathrm{M}_{3}$ at $t=0$ are shown in Figures $26 \mathrm{~b} 26 \mathrm{~d}$ and at $t=6$ in Figures $27 \mathrm{~b} 27 \mathrm{~d}$ (obtained with the NXFEM method).

\subsubsection{Comparison of results}

The penalty parameter $\varepsilon$ for the $\mathrm{FD}$ and $\mathrm{FD}_{\text {stab }}$ methods is $\varepsilon=10^{-4}$ for all levels of refinement. As regards the stabilization parameter $\varepsilon_{\mathrm{M}}$, we have $\varepsilon_{\mathrm{M}}=1$ with $\mathrm{M}_{1}, \varepsilon_{\mathrm{M}}=10^{-1}$ with $\mathrm{M}_{2}$ and $\varepsilon_{\mathrm{M}}=10^{-4}$ with $\mathrm{M}_{3}$. These optimal choices will be discussed in Section 4.3 .3 below.

Figures 28 30 present the snapshots of the fluid velocity magnitude near the vesicle and its

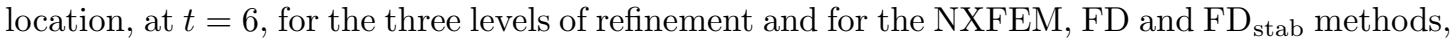
respectively. Note that some slight oscillations in the isolines of the reference ALE solution are visible, which can be related to the distortion of the deformed fluid mesh (see Figure 27a). We can clearly observe that the NXFEM method is able to predict the correct location of the interface even with the coarsest approximation (see Figure 28b). The situation is much more delicate for the FD method which, at all grid levels, shows major mass losses across the interface. In other words, the area of the vesicle significantly evolves during the simulation. This unphysical phenomenon is corrected by the $\mathrm{FD}_{\text {stab }}$ after grid refinement (see Figure 30d). This issue is explicitly measured in Figure 31, which reports the time history of the relative vesicle area for the three unfitted mesh methods. As expected, the area of the vesicle obtained with ALE method is practically constant over time. Note that the reference simulation breaks down at $t=6.0275$, due to the stretching of fluid elements around the interface. Area changes are negligible with the NXFEM method and, after reasonable grid refinement, with the $\mathrm{FD}_{\text {stab }}$ method.

Some pressures snapshots are given in Figures 32,34 respectively for the NXFEM, FD and 


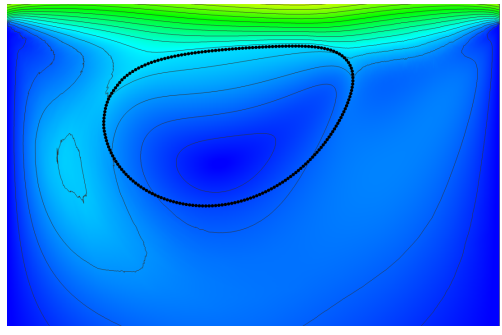

(a) ALE method - Reference.

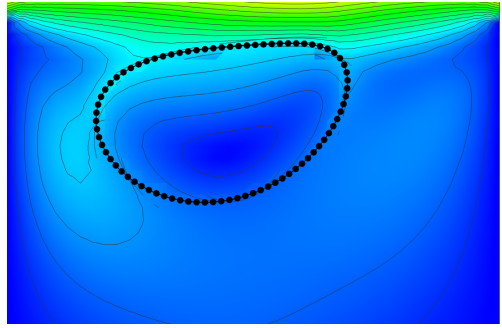

(c) NXFEM method $-\mathrm{M}_{2}$.

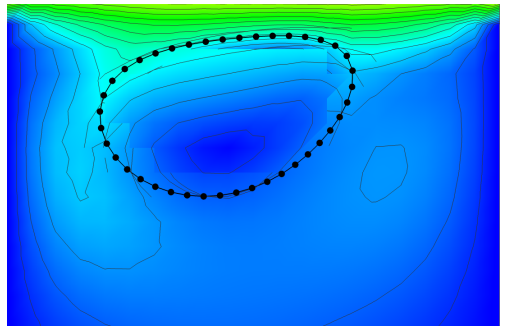

(b) NXFEM method - $M_{1}$.

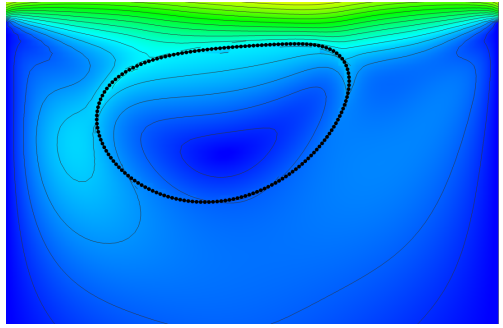

(d) NXFEM method $-M_{3}$.

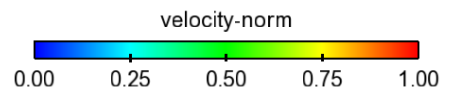

Figure 28: Snapshots of the fluid velocity magnitude at $t=6$ obtained with NXFEM.

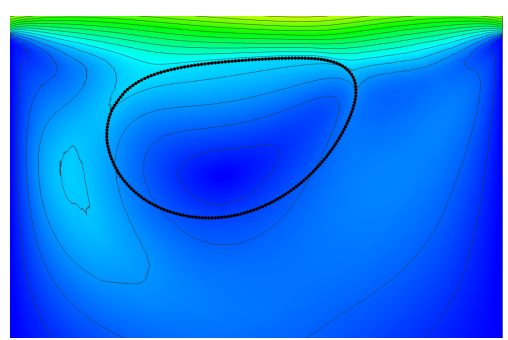

(a) ALE method - Reference.

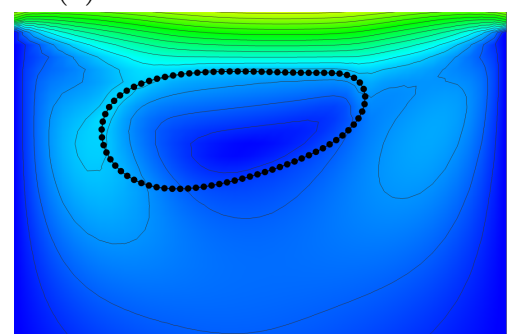

(c) FD method $-\mathrm{M}_{2}$.

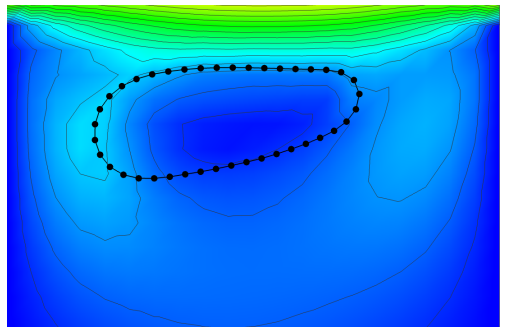

(b) FD method $-M_{1}$.

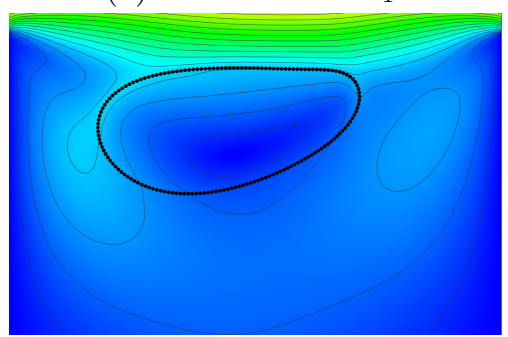

(d) FD method $-\mathrm{M}_{3}$.

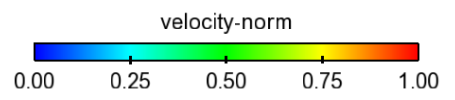

Figure 29: Snapshots of the fluid velocity magnitude at $t=6$ obtained with FD.

$\mathrm{FD}_{\text {stab }}$ methods. Note that the FD method does not predict any significant pressure jump 


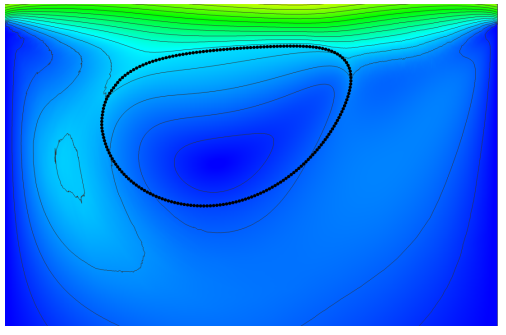

(a) ALE method - Reference.

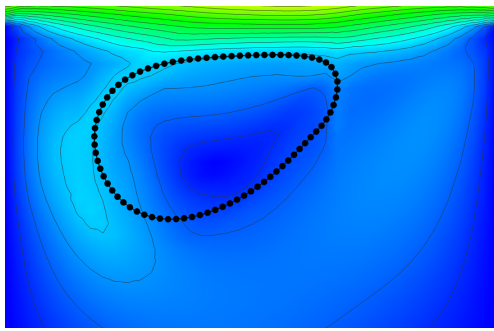

(c) $\mathrm{FD}_{\text {stab }}$ method $-\mathrm{M}_{2}$.

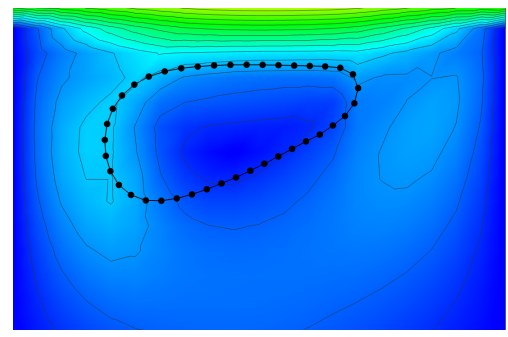

(b) $\mathrm{FD}_{\text {stab }}$ method $-\mathrm{M}_{1}$

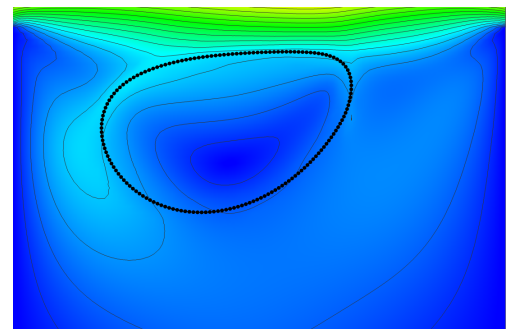

(d) $\mathrm{FD}_{\text {stab }}$ method $-\mathrm{M}_{3}$

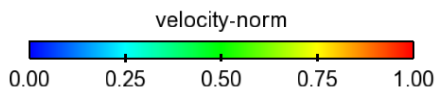

Figure 30: Snapshots of the fluid velocity magnitude at $t=6$ obtained with $\mathrm{FD}_{\text {stab }}$.

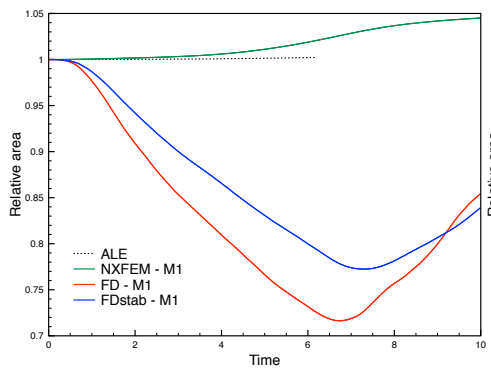

(a) $M_{1}$ vs. Reference.

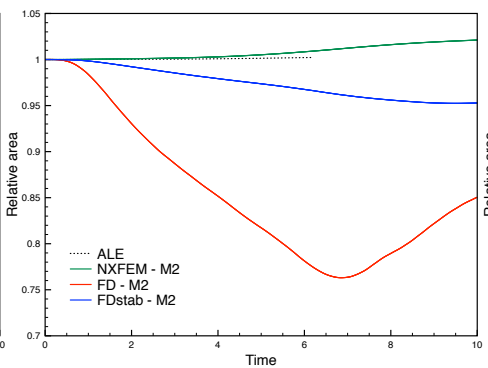

(b) $\mathrm{M}_{2}$ vs. Reference.

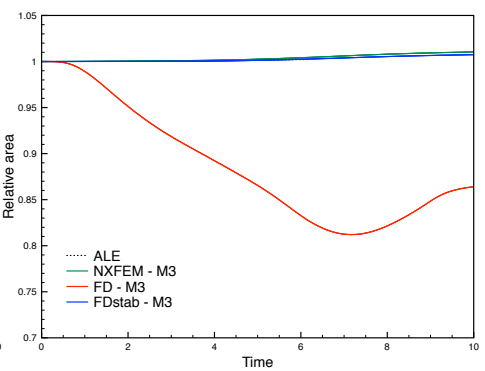

(c) $M_{3}$ vs. Reference.

Figure 31: Time history of the relative area of the vesicle.

between the inner vesicle and the rest of the cavity, no matter what level of refinement is used. On the contrary, the NXFEM method is able to predict it and it also improves after refinement. A similar behavior can be observed from $\mathrm{M}_{2}$ with the $\mathrm{FD}_{\text {stab }}$ variant. Particularly noticeable is the very good agreement between the NXFEM and $\mathrm{FD}_{\text {stab }}$ approximations with the reference solution, for the highest level of refinement.

Similar observations can be made from Figure 35. which reports the time history of the trajectory of the bottom of the vesicle. Again, the FD method presents shifted trajectories compared to the other methods, consequence of the continuous pressure approximation. 


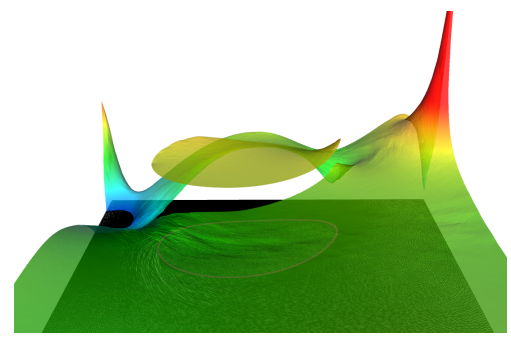

(a) ALE method - Reference.

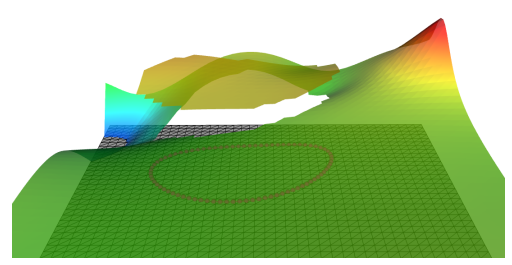

(c) NXFEM method - $\mathrm{M}_{2}$.

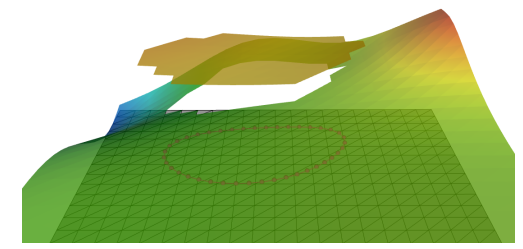

(b) NXFEM method - $\mathrm{M}_{1}$.

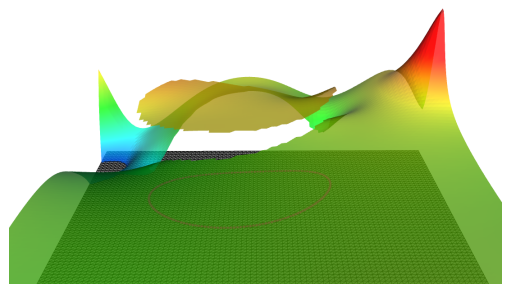

(d) NXFEM method - $\mathrm{M}_{3}$.

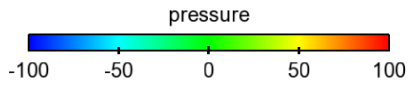

Figure 32: Snapshots of the fluid elevated pressure at $t=6$ obtained with NXFEM.

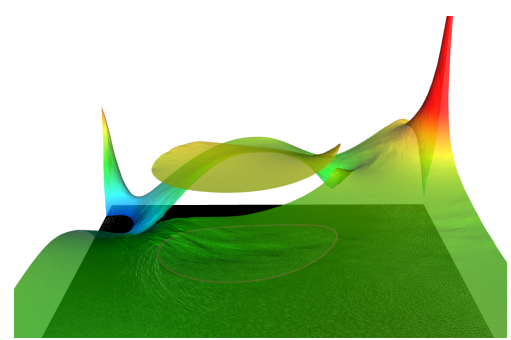

(a) ALE method - Reference.

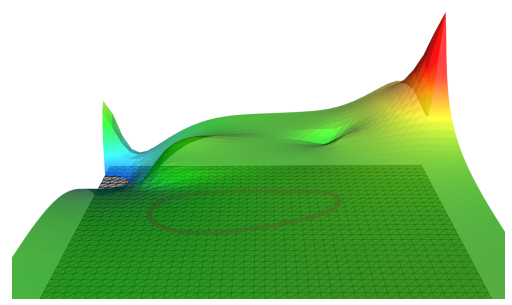

(c) FD method - $\mathrm{M}_{2}$.

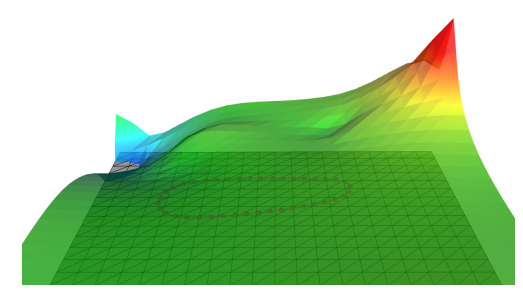

(b) FD method - $\mathrm{M}_{1}$.

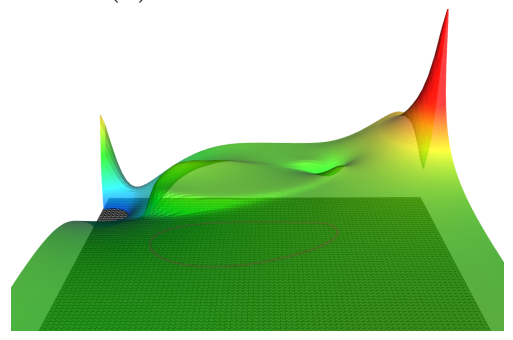

(d) FD method $-\mathrm{M}_{3}$.

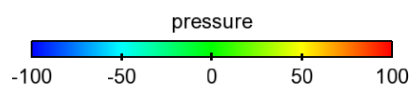

Figure 33: Snapshots of the fluid elevated pressure at $t=6$ obtained with FD. 


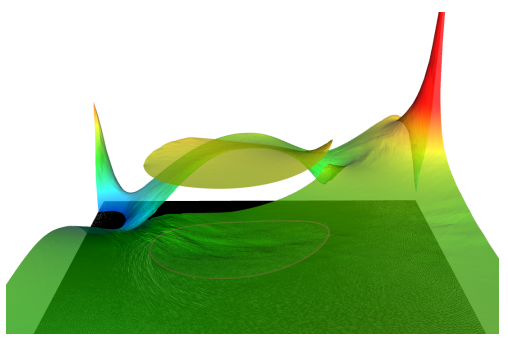

(a) ALE method - Reference.

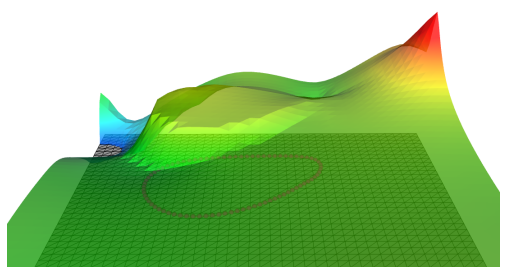

(c) $\mathrm{FD}_{\text {stab }}$ method $-\mathrm{M}_{2}$.

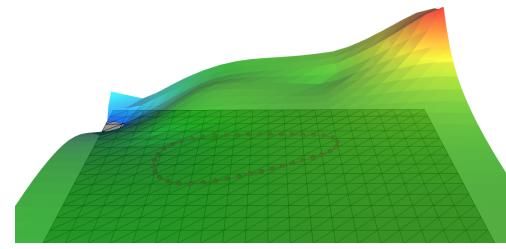

(b) $\mathrm{FD}_{\text {stab }}$ method $-\mathrm{M}_{1}$.

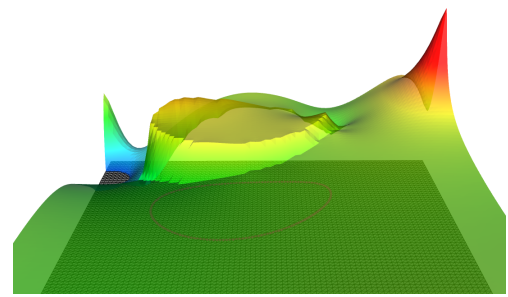

(d) $\mathrm{FD}_{\text {stab }}$ method $-\mathrm{M}_{3}$.

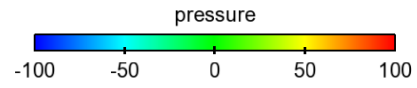

Figure 34: Snapshots of the fluid elevated pressure at $t=6$ obtained with FD $_{\text {stab }}$.

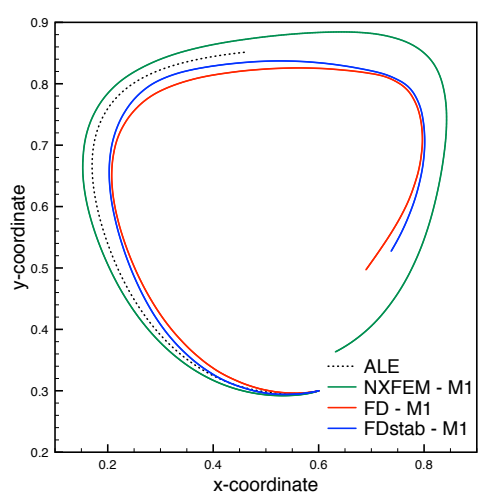

(a) $M_{1}$ vs. Reference.

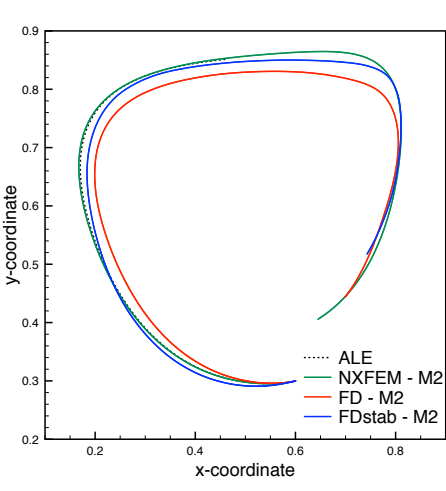

(b) $\mathrm{M}_{2}$ vs. Reference.

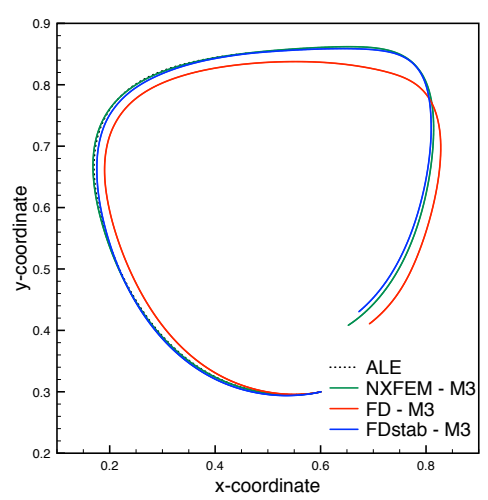

(c) $M_{3}$ vs. Reference.

Figure 35: Time history of the trajectory of the bottom of the vesicle.

\subsubsection{Choice of the penalty parameters for the fictitious domain methods}

As explained in Subsection 4.1.3, we carried on the same convergence study with respect to the penalty parameter $\varepsilon$, appearing in the FD method. The physical value of interest here is the displacement magnitude of the bottom of the vesicle. For each level of refinement, the case has been run several times with a large panel of values for $\varepsilon$. The same behaviors as the ones described in Section 4.1.3 and Section 4.2.3 have also been observed here. The chosen values of $\varepsilon$ are based on the previous mentioned criteria of convergence.

In order to choose the values of $\varepsilon_{\mathrm{M}}$, we carried on the same kind of convergence study as the one described in Subsection 4.2.3. The possible values taken by this parameter are dependent 
on the size of the area where the stabilization is modified and, hence, of the level of refinement. This explains why $\varepsilon_{M}$ is lower for more refined space-time grids.

\section{Conclusions}

Different unfitted mesh methods for immersed FSI have been compared and validated in a series of 2D-benchmarks involving fully non-linear models and large interface deflections. All the methods discussed are based on low-order first-order Lagrange spatial approximations, both in the fluid and in the solid. A priori, the main strengths and weaknesses of the methods can be summarized as follows:

- The FD methods based on Dirac's Lagrange multipliers and globally continuous fluid approximations are very appealing in practice, particularly for 3D applications, since their computer implementation demands relatively simple modifications on existing fluid solvers. The price to pay is the accuracy issues and the condition number degradation, due to penalty terms;

- The Nitsche-XFEM method is based on recent mathematically sound results (fully consistent treatment of the interface coupling, optimal error estimates). However, in practice, this method requires major intrusive modifications on existent fluid solvers, due in particular to the dynamic nature of the cut-FEM methodology, that can be challenging in 3D with non-structured meshes (a recent study in this direction can be found in [55, see also [37, 32]).

The detailed comparisons of Section 4 showed that, for a given mesh refinement, the best accuracy is obtained with the Nitsche-XFEM method. Another salient result of the present study is that, whenever present, the inaccuracies of the FD method are mainly driven by artificial interfacial mass losses, rather than by the sub-optimal treatment of the interface coupling, notably in the case of closed domains. This observation is supported by the very goods results obtained with the $\mathrm{FD}_{\text {stab }}$ variant and a reasonable space-time grid refinement. The main limitation of this approach is the degradation of the stiffness matrix conditioning induced by the grad-div penalty near the interface.

These ill-conditioning issues can be circumvented by considering either divergence-conforming approximations (see [33, 16] within the immersogeometric framework) or globally discontinuous pressures with higher order polynomials for the velocities (see [4, 8, 7, 10] within the finite element framework). We hence believe that an interesting extension of this work could be the development of alternative fictitious domain methods with enhanced interfacial mass conservation which preserve, for convenience, the low-order nature of the finite element fluid approximations. In particular, we will explore the use of $\mathbb{P}_{1} / \mathbb{P}_{0}$ stabilized approximations with a pressure stabilization coefficient which vanishes within a $h$-dependent vicinity of the interface, in order to remove the inconsistency of the pressure jumps.

\section{Acknowledgements}

The research leading to these results has been partially supported by the project MIVANA. MIVANA is a collaborative project for the development of new technologies for mitral valve repair. It is led by the start-up company Kephalios, with the participation of the start-up company Epygon, and it has received funds from the French government, in the context of the program "Investissement d'Avenir". 


\section{References}

[1] F. Alauzet. A changing-topology moving mesh technique for large displacements. Eng. Comput., 30(2):175-200, 2014.

[2] F. Alauzet, B. Fabrèges, M. A. Fernández, and M. Landajuela. Nitsche-XFEM for the coupling of an incompressible fluid with immersed thin-walled structures. Comput. Methods Appl. Mech. Engrg., 301:300-335, 2016.

[3] M. Astorino, J.-F. Gerbeau, O. Pantz, and K.-F. Traoré. Fluid-structure interaction and multi-body contact: application to aortic valves. Comput. Methods Appl. Mech. Engrg., 198(45-46):3603-3612, 2009.

[4] F. Baaijens. A fictitious domain/mortar element method for fluid-structure interaction. Int. Jour. Num. Meth. Fluids, 35:743-761, 2001.

[5] Y. Bao, A. Donev, B.E. Griffith, D.M. McQueen, and C.S. Peskin. An immersed boundary method with divergence-free velocity interpolation and force spreading. J. Comput. Phys., 347:183-206, 2017.

[6] K.J. Bathe. Finite Element Procedures. Prentice Hall, 1996.

[7] D. Boffi, N. Cavallini, F. Gardini, and L. Gastaldi. Local mass conservation of Stokes finite elements. J. Sci. Comput., 52(2):383-400, 2012.

[8] D. Boffi, N. Cavallini, F. Gardini, and L. Gastaldi. Stabilized Stokes elements and local mass conservation. Boll. Unione Mat. Ital. (9), 5(3):543-573, 2012.

[9] D. Boffi, N. Cavallini, and L. Gastaldi. Finite element approach to immersed boundary method with different fluid and solid densities. Math. Models Methods Appl. Sci., 21(12):2523-2550, 2011.

[10] Daniele Boffi, Nicola Cavallini, and Lucia Gastaldi. The finite element immersed boundary method with distributed lagrange multiplier. SIAM Journal on Numerical Analysis, $53(6): 2584-2604,2015$.

[11] Daniele Boffi and Lucia Gastaldi. A fictitious domain approach with lagrange multiplier for fluid-structure interactions. Numer. Math., 135(3):711-732, 2017.

[12] E. Burman. Ghost penalty. C. R. Math. Acad. Sci. Paris, 348(21-22):1217-1220, 2010.

[13] E. Burman and M. A. Fernández. An unfitted Nitsche method for incompressible fluidstructure interaction using overlapping meshes. Comput. Methods Appl. Mech. Engrg., 279:497-514, 2014.

[14] E. Burman, M. A. Fernández, and P. Hansbo. Continuous interior penalty finite element method for Oseen's equations. SIAM J. Numer. Anal., 44(3):1248-1274, 2006.

[15] H. Casquero, C. Bona-Casas, and H. Gomez. NURBS-based numerical proxies for red blood cells and circulating tumor cells in microscale blood flow. Comput. Methods Appl. Mech. Engrg., 316:646-667, 2017.

[16] H. Casquero, C. Bona-Casas, H. Gomez, and Y. Zhang. Divergence-conforming and fullyimplicit simulation of microscale blood flow. In International Conference on Isogeometric Analysis (IGA 2017), Pavia (Italy), September 2017. 
[17] D. Chapelle and K.J. Bathe. The Finite Element Analysis of Shells - Fundamentals. Springer, 2011. 2nd Edition.

[18] D. Chapelle and A. Ferent. Modeling of the inclusion of a reinforcing sheet within a 3D medium. Math. Models Methods Appl. Sci., 13(4):573-595, 2003.

[19] G.-H. Cottet, E. Maitre, and T. Milcent. Eulerian formulation and level set models for incompressible fluid-structure interaction. M2AN Math. Model. Numer. Anal., 42(3):471492, 2008.

[20] J. De Hart, G. W M Peters, P. J G Schreurs, and F. P T Baaijens. A three-dimensional computational analysis of fluid-structure interaction in the aortic valve. Journal of Biomechanics, 36(1):103-112, 2003.

[21] N Diniz Dos Santos, J-F Gerbeau, and J-F Bourgat. A partitioned fluid-structure algorithm for elastic thin valves with contact. Computer Methods in Applied Mechanics and Engineering, 197(19):1750-1761, 2008.

[22] Boyce E. Griffith and Xiaoyu Luo. Hybrid finite difference/finite element immersed boundary method. International Journal for Numerical Methods in Biomedical Engineering, 33(12):e2888-n/a, 2017.

[23] B. Fabrèges and B. Maury. Approximation of single layer distributions by dirac masses in finite element computations. Journal of Scientific Computing, 58(1):25-40, 2014.

[24] M. A. Fernández and J.-F. Gerbeau. Algorithms for fluid-structure interaction problems. In Cardiovascular mathematics, volume 1 of MS\&A. Model. Simul. Appl., pages 307-346. Springer, 2009.

[25] K.J. Galvin, A. Linke, L.G. Rebholz, and N.E. Wilson. Stabilizing poor mass conservation in incompressible flow problems with large irrotational forcing and application to thermal convection. Comput. Methods Appl. Mech. Engrg., 237/240:166-176, 2012.

[26] A. Gerstenberger and W.A. Wall. An extended finite element method/Lagrange multiplier based approach for fluid-structure interaction. Comput. Methods Appl. Mech. Engrg., 197(19-20):1699-1714, 2008.

[27] A. J. Gil, A. Arranz Carreño, J. Bonet, and O. Hassan. An enhanced Immersed Structural Potential Method for fluid-structure interaction. Journal of Computational Physics, 250:178-205, 2013.

[28] R. Glowinski, T.W. Pan, T.I. Hesla, and D.D. Joseph. A distributed Lagrange mutiplier/fictitious domain method for particulate flows. Int. J. of Multiphase Flow, 25:755-794, 1999.

[29] Boyce E. Griffith. On the volume conservation of the immersed boundary method. Communications in Computational Physics, 12(2):401-432, 2012.

[30] E. Hachem, S. Feghali, R. Codina, and T. Coupez. Immersed stress method for fluidstructure interaction using anisotropic mesh adaptation. Internat. J. Numer. Methods Engrg., 94(9):805-825, 2013.

[31] C. Hesch, A. J. Gil, A. Arranz Carreño, and J. Bonet. On continuum immersed strategies for Fluid-Structure Interaction. Computer Methods in Applied Mechanics and Engineering, 247-248:51-64, 2012. 
[32] C. Kadapa, W.G. Dettmer, and D. Perić. A stabilised immersed framework on hierarchical bspline grids for fluid-flexible structure interaction with solid-solid contact. Comput. Methods Appl. Mech. Engrg., 335:472-489, 2018.

[33] D. Kamensky, Y. Hsu, M.-C. dnd Yu, Evans. J.A., M.S. Sacks, and T.J.R. Hughes. Immersogeometric cardiovascular fluid-structure interaction analysis with divergence-conforming B-splines. Comput. Methods Appl. Mech. Engrg., 314:408-472, 2017.

[34] David Kamensky, Ming-Chen Hsu, Dominik Schillinger, John A Evans, Ankush Aggarwal, Yuri Bazilevs, Michael S Sacks, and Thomas JR Hughes. An immersogeometric variational framework for fluid-structure interaction: Application to bioprosthetic heart valves. Computer methods in applied mechanics and engineering, 284:1005-1053, 2015.

[35] David Kamensky, Ming-Chen Hsu, Yue Yu, John A Evans, Michael S Sacks, and Thomas JR Hughes. Immersogeometric cardiovascular fluid-structure interaction analysis with divergence-conforming b-splines. Computer methods in applied mechanics and engineering, 314:408-472, 2017.

[36] Mikel Landajuela, Marina Vidrascu, Dominique Chapelle, and Miguel A. Fernández. Coupling schemes for the FSI forward predication challenge: comparative study and validation. Int. J. Numer. Methods Biomed. Eng., 33(4):e02813, 23, 2017.

[37] André Massing, Mats G. Larson, and Anders Logg. Efficient implementation of finite element methods on nonmatching and overlapping meshes in three dimensions. SIAM Journal on Scientific Computing, 35(1):C23-C47, 2013.

[38] U.K. Müller, A. Wasim, E. Fontaine, O. Berg, Y. Cao, D. Lentink, S. Kranenbarg, and J.L. van Leeuwen. Fish and Flag - Exploring Fluid-Structure Interaction during Undulatory Swimming in Fish. In C.T. Lim and J.C.H. Goh, editors, 6th World Congress of Biomechanics (WCB 2010). August 1-6, 2010 Singapore, volume 31 of IFMBE Proceedings, pages 44-47. Springer, 2010.

[39] A. Patel. Lagrange multiplier method with penalty for elliptic and parabolic interface problems. J. Appl. Math. Comput., 37(1-2):37-56, 2011.

[40] C.S. Peskin. The immersed boundary method. Acta Numer., 11:479-517, 2002.

[41] C.S. Peskin and B.F. Printz. Improved volume conservation in the computation of flows with immersed elastic boundaries. J. Comput. Phys., 105(1):33-46, 1993.

[42] Isabelle Ramière, Philippe Angot, and Michel Belliard. A fictitious domain approach with spread interface for elliptic problems with general boundary conditions. Computer Methods in Applied Mechanics and Engineering, 196(4-6):766-781, 2007.

[43] T. Richter. A fully eulerian formulation for fluid-structure-interaction problems. J. Comput. Phys., 233:227-240, 2013.

[44] Saswati Roy, Luca Heltai, and Francesco Costanzo. Benchmarking the immersed finite element method for fluid-structure interaction problems. Computers and Mathematics with Applications, 69(10):1167-1188, 2015.

[45] T. Sawada and A. Tezuka. LLM and X-FEM based interface modeling of fluid-thin structure interactions on a non-interface-fitted mesh. Comput. Mech., 48(3):319-332, 2011. 
[46] K. Stein, T. Tezduyar, and R. Benney. Mesh moving techniques for fluid-structure interactions with large displacements. Journal of Applied Mechanics, 70(1):58-63, 2003.

[47] T.E. Tezduyar. Stabilized finite element formulations for incompressible flow computations. In Advances in applied mechanics, Vol. 28, volume 28 of Adv. Appl. Mech., pages 1-44. Academic Press, Boston, MA, 1992.

[48] F.-B. Tian, H. Dai, H. Luo, J.F. Doyle, and B. Rousseau. Fluid-structure interaction involving large deformations: 3D simulations and applications to biological systems. J. Comput. Phys., 258:451-469, 2014.

[49] R. van Loon, P. D. Anderson, J. de Hart, and F. P T Baaijens. A combined fictitious domain/adaptive meshing method for fluid-structure interaction in heart valves. International Journal for Numerical Methods in Fluids, 46(5):533-544, 2004.

[50] Xingshi Wang and Lucy T. Zhang. Interpolation functions in the immersed boundary and finite element methods. Computational Mechanics, 45(4):321-334, 2010.

[51] T. Wick. Fluid-structure interactions using different mesh motion techniques. Comp. \& Struct., 89(13-14):1456-1467, 2011.

[52] Thomas Wick. Flapping and contact FSI computations with the fluid-solid interfacetracking/interface-capturing technique and mesh adaptivity. Computational Mechanics, 53(1):29-43, 2014.

[53] Guanyu Zhou and Norikazu Saito. Analysis of the fictitious domain method with penalty for elliptic problems. Japan Journal of Industrial and Applied Mathematics, 31(1):57-85, 2014.

[54] A. Zilian and A. Legay. The enriched space-time finite element method (EST) for simultaneous solution of fluid-structure interaction. Internat. J. Numer. Methods Engrg., 75(3):305$334,2008$.

[55] S. Zonca, S. Vergara, and L. Formaggia. An unfitted formulation for the interaction of an incompressible fluid with a thick structure via an XFEM/DG approach. SIAM J. Sci. Comput., 40(1):B59-B84, 2018. 


\section{Contents}

\begin{tabular}{lll}
\hline & Introduction & 3
\end{tabular}

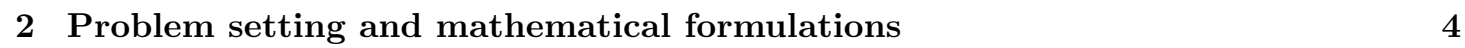

2.1 Geometric configurations and notation . . . . . . . . . . . . . . . . . . . . 4

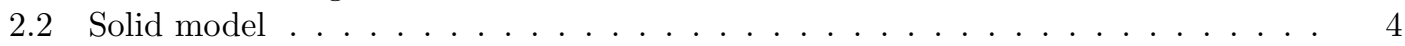

2.3 Coupled problem with Eulerian formalism in the fluid . . . . . . . . . . . . . . 5

2.4 Coupled problem with ALE formalism in the fluid . . . . . . . . . . . . . . . 6

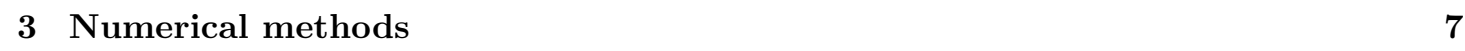

3.1 Fitted mesh methods . . . . . . . . . . . . . . . . . . . . . . . 7

3.2 Unfitted mesh methods . . . . . . . . . . . . . . . . . . . . . . . . 9

3.2.1 Fictitious domain method . . . . . . . . . . . . . . . . . . . . . . . . . . . . . . . . . . . . . .

3.2 .2 Nitsche-XFEM method . . . . . . . . . . . . . . . . . . . . 11

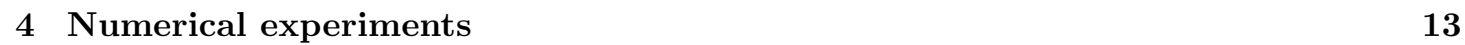

4.1 Example 1. Open valve . . . . . . . . . . . . . . . . . . . . . 13

4.1 .1 Spatial and temporal discretization . . . . . . . . . . . . . . . . . . 14

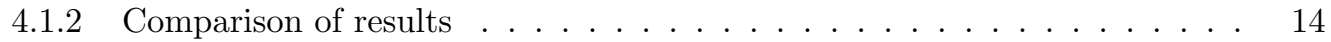

4.1.3 Choice of the penalty parameters for the fictitious domain methods. . . . 18

4.2 Example 2. Closed valve . . . . . . . . . . . . . . . . . . . . . . . . . . 19

4.2.1 Spatial and temporal discretization . . . . . . . . . . . . . . . . . . . . . . . . . . . 20

4.2 .2 Comparison of results . . . . . . . . . . . . . . . . . 20

4.2.3 Choice of the penalty parameters for the fictitious domain methods. . . . 25

4.3 Example 3. Vesicle in lid-driven cavity flow . . . . . . . . . . . . . . . . . 25

4.3 .1 Spatial and temporal discretization . . . . . . . . . . . . . . . . 26

4.3 .2 Comparison of results . . . . . . . . . . . . . . . . 27

4.3.3 Choice of the penalty parameters for the fictitious domain methods. . . . 31

\begin{tabular}{|lr}
\hline 5 & Conclusions \\
\hline
\end{tabular} 


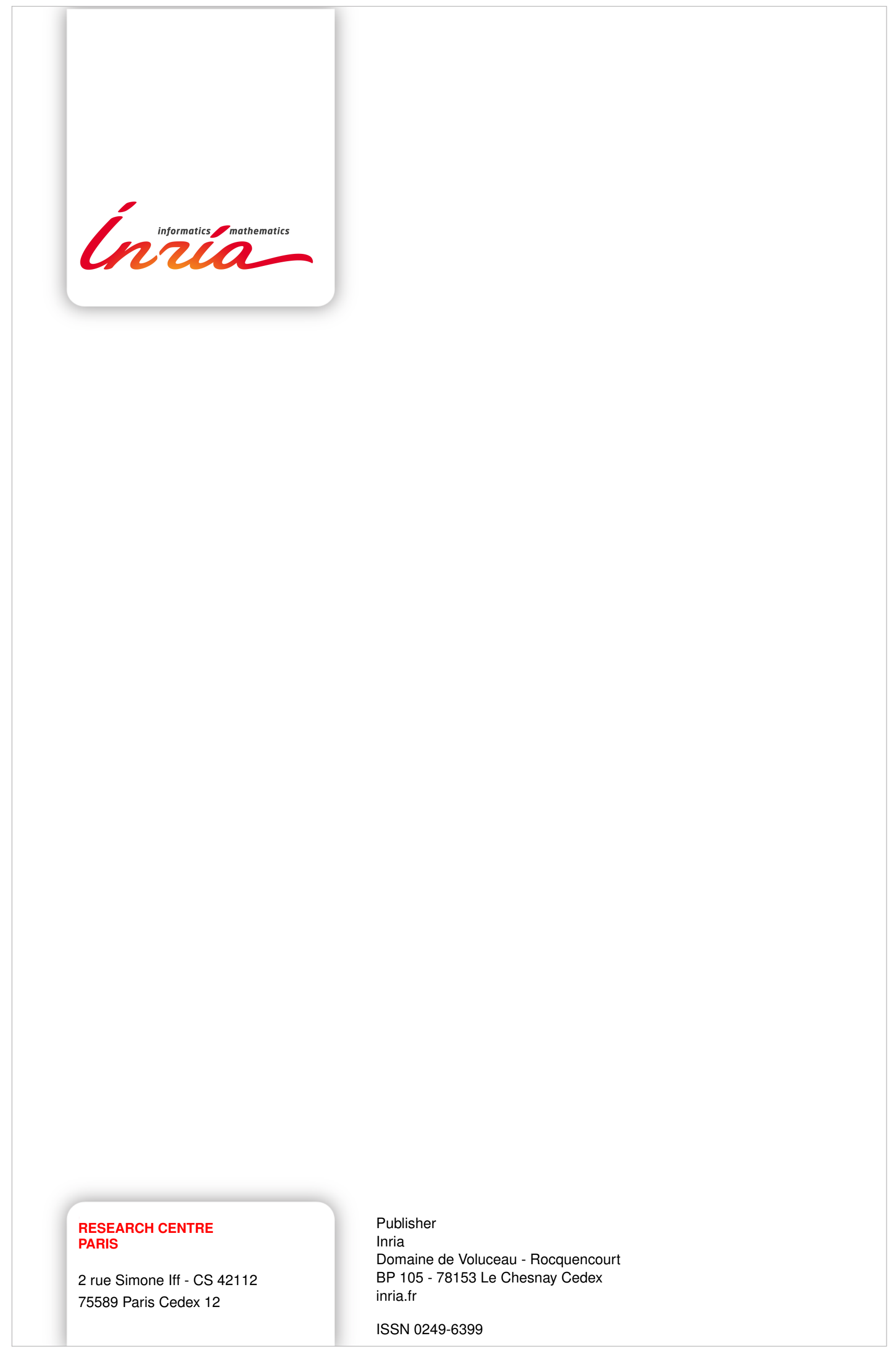

